\title{
一氧化氮供体化合物的合成方法研究进展
}

$$
\begin{aligned}
& \text { 王 兵 }{ }^{a, b} \text { 李 娜 }{ }^{a, b} \text { 刘 腾 }{ }^{a, b} \text { 王英爱 }{ }^{a, b} \\
& \text { 王晓静*, } a, b \text { 孙 捷*, }, a, b
\end{aligned}
$$

( ${ }^{a}$ 济南大学 山东省医学科学院 医学与生命科学学院 济南 250200) ${ }^{b}$ 山东省医学科学院药物研究所 国家卫生部生物技术药物重点实验室 山东省罕少见病重点实验室 济南 250062)

摘要 一氧化氮(NO)作为生物信使或效应分子在体内发挥重要的生理作用, 因其具有多种多样的生物活性在临床应用 方面得到广泛关注. 体内 NO 生成不足常与多种疾病的形成密切相关, NO 供体化合物可以通过在体内释放 NO 治疗和 预防多种疾病. 随着 NO 供体化合物的广泛应用, 其合成方法引起了国内外研究人员的高度重视. 结合国内外学者对这 方面的研究，对近 10 年 NO 供体化合物的合成方法研究进展进行综述.

关键词 一氧化氮供体; 合成方法; 药理活性

\section{Research Progress on Synthesis of Nitric Oxide Donor Compounds}

\author{
Wang, Bing ${ }^{a, b} \quad \mathrm{Li}, \mathrm{Na}^{a, b} \quad$ Liu, Teng $^{a,} \quad$ Wang, Ying'ai ${ }^{a, b}$ \\ Wang, Xiaojing ${ }^{*, a, b} \quad$ Sun, Jie $\mathrm{e}^{*, a, b}$
}

( ${ }^{a}$ School of Medicine and Life Sciences, University of Jinan-Shandong Academy of Medical Sciences, Jinan 250200)

$\left({ }^{b}\right.$ Key Laboratory for Biotech-Drugs Ministry of Health, Key Laboratory for Rare \& Uncommon Diseases of Shandong Province, Institute of Materia Medica, Shandong Academy of Medical Sciences, Jinan 250062)

\begin{abstract}
Nitric oxide as a biological messenger or effector molecule plays an important physiological role in the body. Owing to its various biological activities, it has received wide attention in clinical practice. Insufficient NO production in vivo is closely related with a variety of diseases. NO donor compounds can release NO in vivo to treat and prevent many diseases. With its wide application in medicine, the methods for the synthesis of NO donor compounds have attracted much attention of researchers. In this paper, the recent advances in the past 10 years in synthetic methods for NO donor compounds are reviewed. Keywords nitric oxide donor; synthetic method; pharmacological activity
\end{abstract}

近几十年, NO 常被人们用以阐明许多过去未能解 释的生理现象, 成为近年来研究的热点. NO 是一种寿 命较短的自由基, 半衰期仅数秒钟, 由于其分子小且具 有亲脂性, 所以很容易透过细胞膜 ${ }^{[1]}$. 在体内由 $L$-精氨 酸和氧分子在一氧化氮合酶(NOS)催化下生成 ${ }^{[2]}$. NO 在 哺乳动物生理和病理生理中发挥着非常重要的作用 ${ }^{[3]}$, 如参与维持微血管和大血管的动态平衡 ${ }^{[4]}$ 、神经信号传

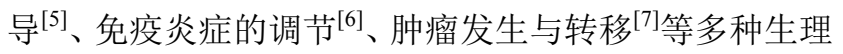
病理过程. 体内 $\mathrm{NO}$ 生成不足常与多种疾病的形成密切 相关 ${ }^{[8 \sim 11]}$, 因此外源性 NO 对于这些疾病的预防和治疗
有着重要意义 ${ }^{[12]}$. 所以, NO 供体药物成为了热门的新 型药物研究对象. NO 供体是指一类在体内经简单酶解 或非酶作用后释放出 $\mathrm{NO}$ 的化合物，是 $\mathrm{NO}$ 的储运形式， 可以克服 $\mathrm{NO}$ 本身难携带、难定量、半衰期短 ${ }^{[13]}$ 等缺点. $\mathrm{NO}$ 供体药物主要是指 $\mathrm{NO}$ 供体与已知功效的药物通过 某些基团连接起来形成的具有协同生物活性的新化合 物 ${ }^{[14,15]}$. 目前的研究趋势是利用前药原理, 将已知药物 或已知活性化合物的结构与各类 NO 供体通过各种连接 基团结合而制成前药. 这种前药可在体内经相关酶或非 酶作用释放原药和 NO, 使其分别发挥各自作用. 目前

\footnotetext{
* Corresponding author. E-mail: xiaojing6@gmail.com; 635775299@qq.com

Received October 21, 2016; revised December 29, 2016; published online January 20, 2017.

Project supported by the Natural Science Foundation of Shandong Province (No. ZR2015YL041).

山东省自然科学基金(No. ZR2015YL041)资助项目.
} 
在研究的 NO 供体主要有硝酸酯类、呋咱氮氧化物类、 偶氮二醇烯鎓盐类、肜类、NO-金属配合物类、S-亚硝 基硫醇类、斯德酮亚胺类、胍类、羟胺类及 $N$-羟基艮类 等 ${ }^{[16]}$. 其中硝酸酯类、呋咱氮氧化物类和偶氮二醇烯鎓 盐类 NO 供体是近年来的研究热点. 药理研究证明, 很 多 NO 供体药物的药理活性比原药好, 而且不良反应显 著低于原药 ${ }^{[17]}$, 同时这类药物还具有提高药物的生物 利用度、减小毒副作用、增加药物稳定性、促使药物长 效化 ${ }^{[18]}$ 等优点.

NO 供体类化合物多种活性的不断发现及应用范围 的不断拓展已经引起了国内外很多有机合成研究人员 的高度重视. 通常化学方法合成 NO 供体类化合物主要 包括两个关键点: (1)如何构建 NO 供体化合物的 NO 释 放基团; (2)如何将 NO 释放基团准确地与已知功效的药 物连接. 本文总结了国内外学者对这方面的相关研究, 对 NO 供体类化合物的合成方法进行综述.

\section{1 硝酸酯类 NO 供体的合成}

硝酸酯类 NO 供体是一类经典药物, 硝酸甘油作为 治疗心绞痛的药物已经应用了很多年. 这类 NO 供体药 物结构简单、易于合成、应用比较普遍 ${ }^{[19]}$. 目前硝酸酯 类 NO 供体药物的合成主要有以下几种合成路线. 通过 酯键将 NO 供体和药物连接, 通过醚键将 NO 供体和药 物连接以及通过肽键连接.

\section{1 酯化反应}

将带有 NO 供体基团的分子片段与化合物通过酯键 连接，该方法条件温和，易于操作，适合用于多数带有 羧基的化合物与 NO 供体基团相连.

2007 年, López 等 ${ }^{[20]}$ 合成了带有 NO 供体基团的生 育酚类似物. 该反应通过成酯反应将带有 NO 供体基团 的醇与生育酚类似物连接起来(Eq. 1). 此法适用于多数 带有羧基的化合物与 NO 供体基团相连.

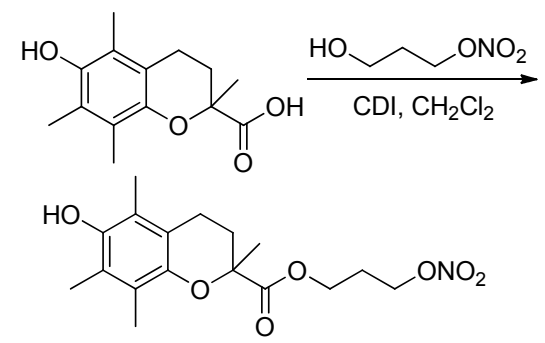

2009 年, Abdel-Hafez 等 ${ }^{[21]}$ 合成了一系列具有抗菌 抗炎活性的 NO 供体化合物. 该反应首先进行酰氯的醇 解合成溴代产物, 再用硝酸盐进行硝化获得有机硝酸酯 类产物(Scheme 1). 药理研究表明, 该化合物比原药具 有更强的抗炎活性, 而且致溃疡能力明显减弱.

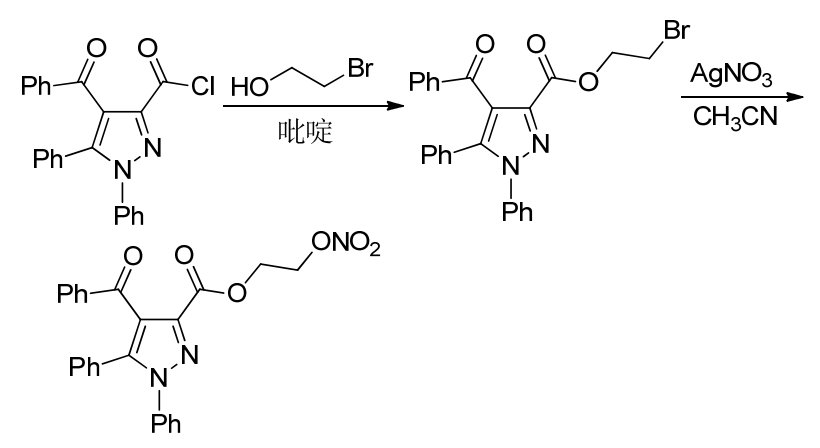

图式 1 带有 NO 供体的吡唑-3-羧酸衍生物的合成路线 Scheme 1 Synthetic routes of pyrazole-3-carboxylic acid derivatives bearing $\mathrm{NO}$ donor

2009 年, Abdellatif 等 ${ }^{[17]}$ 报道在非甾体类抗炎药骨架 上添加 NO 供体可以减轻非甾体类抗炎药的胃肠道副作 用. 该反应是先将非甾体类抗炎药的羧基酰氯化然后再 通过成酯反应将 NO 供体与药物偶联(Scheme 2). 该合成 方案通过将着基酰氯化使整个成酯反应更加容易进行. 药理研究表明, 该化合物具有与原药相当的抗炎活性, 而且可以降低非甾体类抗炎药的胃肠道不良反应.

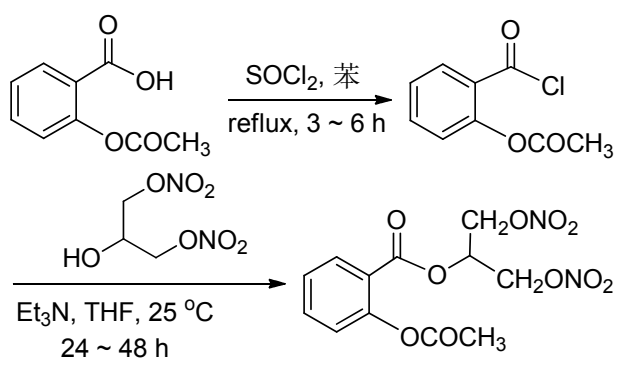

图式 2 阿司匹林和 NO 供体化合物参与的偶联反应 Scheme 2 Cross-coupling reactions of aspirin and nitric oxide donor compound

2012 年, Rolando 等 ${ }^{[22]}$ 合成了带有 NO 供体基团的 依达拉奉衍生物，依达拉奉是自由基清除剂，与 NO 供 体基团偶联后可增强其抗氧化作用. 该方法先用带有 $\mathrm{NO}$ 供体基团的羧酸进行酰氯化反应，再和药物依达拉 奉进行成酯反应获得带有 NO 供体基团的依达拉奉衍生 物(Scheme 3).

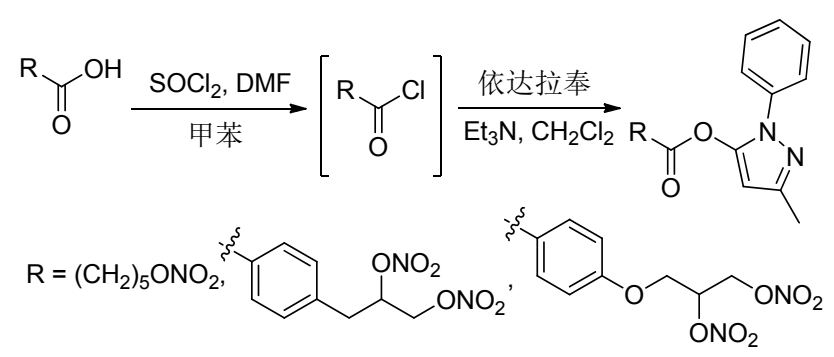

图式 3 带有 NO 供体的依达拉奉衍生物的合成路线

Scheme 3 Synthetic routes of edaravone derivatives bearing NO donor 
2015 年, Digiacomo 等 ${ }^{[23]}$ 合成了系列带有 NO 供体 基团的胰岛素促分泌素衍生物. 该方案直接将胰岛素促 分泌素药物瑞格列奈和那格列奈与带有 NO 供体基团的 醇类化合物通过成酯反应连接起来(Scheme 4). 药理实 验证明这类化合物不仅可以降低血糖还具有血管保护 作用, 可以用来治疗 II 型糖尿病及其引起的血管并发 症.

\section{2 酰胺化反应}

2008 年, Konter 等 ${ }^{[24]}$ 合成了一种带有 NO 供体基团 的抗菌药酮康坐类似物. 该反应是先进行酰胺的水解反 应再进行酰化反应形成带有 NO 供体基团的抗菌化合物 (Eq. 2). 该方法可用于氨类化合物的 NO 供体连接. 药
理研究表明，该化合物的抗真菌活性比原药酮康唑更 强.

2012 年, Bertinaria 等 ${ }^{[25]}$ 合成了系列具有 NO 释放能 力的肌肽类似物 NO 供体(Eq. 3). 该反应可以一次引入 两个 NO 供体基团, 能够增强 NO 的释放. 经药理研究 表明此类化合物对治疗 NO 缺少引起的慢性血管病和神 经疾病具有较好的效果.

2014 年, Biava 等 ${ }^{[26]}$ 通过酰化反应合成了一系列具 有高度选择性的 COX-2 抑制剂(Scheme 5). 该反应通过 酰胺化将羧基与带有 NO 供体的氨基(Eq. 4)直接进行偶 合，简化了反应历程. 其中 NO 供体基团是由氨基醇经 发烟硝酸处理后硝化得到.<smiles>CCOc1cc(CC(=O)NC(CC(C)C)c2ccccc2N2CCCCC2)ccc1C(=O)O</smiles><smiles></smiles><smiles></smiles><smiles></smiles><smiles>CC(=O)OCc1ccc(O[N+]([O-])([O-])[O-])cc1</smiles><smiles>CC(C)C1CCC(C(=O)N[C@@H](Cc2ccccc2)C(=O)OCc2cccc(O[N+](=O)[O-])c2)CC1</smiles>

图式 4 带有 NO 供体的胰岛素促分泌素衍生物的合成路线

Scheme 4 Synthetic routes of insulin-secretagogue derivatives bearing NO donor<smiles>CC(C)(CO[N+](=O)[O-])C(=O)N1CCN(c2ccc(OC[C@H]3CO[C@](Cn4ccnc4)(c4ccc(Cl)cc4Cl)O3)cc2)CC1</smiles><smiles>CCn1cnc(CC(NC(C)C)C(=O)O)c1</smiles><smiles>CCO[C@H](CN)CO[N+](=O)[O-]</smiles><smiles>CC(C)n1cnc(CC(NC=CC(=O)O[N+](=O)[O-])C(=O)NC[C@H](CO[N+](=O)[O-])O[N+](=O)[O-])c1</smiles>

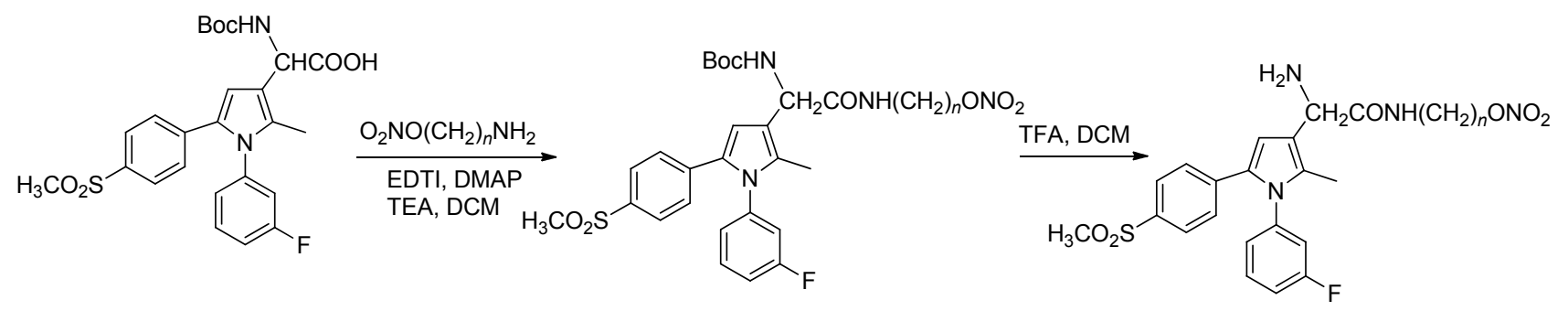

图式 5 带有 NO 供体的 COX-2 抑制剂的合成路线

Scheme 5 Synthetic routes of COX-2 inhibitors bearing NO donor 


$$
\mathrm{HO}\left(\mathrm{CH}_{2}\right)_{n} \mathrm{NH}_{2} \underset{(2)\left(\mathrm{CH}_{3} \mathrm{CO}\right)_{2} \mathrm{O}}{\stackrel{(1)}{\text { 发烟 } \mathrm{HNO}_{3}, \mathrm{DCM}}} \mathrm{O}_{2} \mathrm{NO}\left(\mathrm{CH}_{2}\right)_{n} \mathrm{NH}_{2}
$$

该方法得到的化合物通过药理实验证明具有很强 的抗痛觉过敏和抗水肿作用.

\section{3 取代反应}

\subsection{1 $\mathrm{AgNO}_{3}$ 取代}

硝酸银与卤代烃亲核取代是目前 NO 供体制备的主 要方法, 该方法是先在药物母体上创建卤代基团, 然后 再与 $\mathrm{AgNO}_{3}$ 发生亲核取代反应, 除去生成的卤化银沉 淀即可得到带有硝酸酯类 NO 供体的药物(Scheme 6). 该方法具有广泛的应用范围.

$$
\mathrm{R}-\mathrm{OH} \stackrel{\mathrm{Br}\left(\mathrm{CH}_{2}\right)_{n} \mathrm{Br}}{\longrightarrow} \mathrm{R}-\mathrm{O}\left(\mathrm{CH}_{2}\right)_{n} \mathrm{Br} \underset{\mathrm{CH}_{3} \mathrm{CN}}{\stackrel{\mathrm{AgNO}_{3}}{\longrightarrow}} \mathrm{R}-\mathrm{O}\left(\mathrm{CH}_{2}\right)_{n} \mathrm{ONO}_{2}
$$

图式 6 硝酸银法制备 NO 供体化合物的合成路线 Scheme 6 Synthetic routes of NO-donor compounds by silver nitrate process

2009 年, Liu 等 ${ }^{[27]}$ 合成了在肿瘤细胞定位释放的 NO 供体化合物. 由于卟啉具有肿瘤细胞定位作用, 此类化 合物的合成为靶向释放 NO 提供了重要的参考. 此反应 先利用 1,3-二溴丙烷将对着基苯甲醛的羟基氢取代，再 用 $\mathrm{AgNO}_{3}$ 处理进行亲核取代反应获得带有 $\mathrm{NO}$ 供体的中 间体，再进行叶啉的合成步骤即可合成带有 NO 供体的 卟啉化合物(Scheme 7). 药理研究表明, 该化合物不仅具 有与氟尿嘧啶类似的抗癌活性, 而且可以靶向释放.

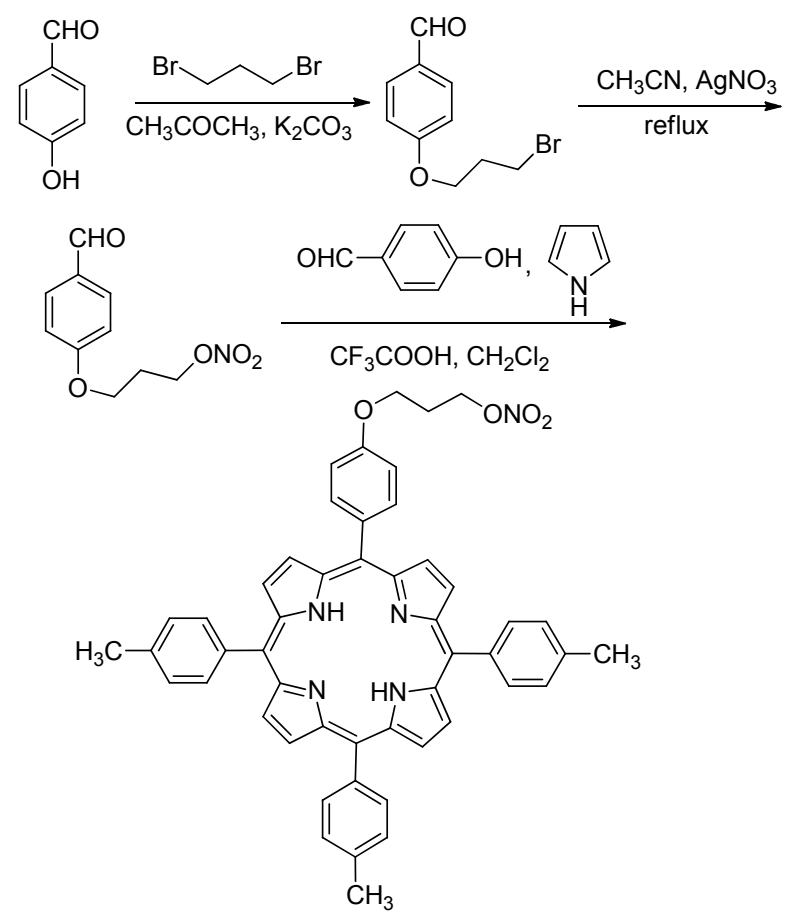

图式 7 带有 NO 供体的卟啉化合物的合成路线

Scheme 7 Synthetic routes of porphyrins bearing NO donor
2009 年, Peng 等 ${ }^{[28]}$ 合成了带有 NO 供体基团的白杨 素衍生物. 该方法先用二溴代烷进行亲核取代反应将溴 代烷基连接到白杨素衍生物骨架上，再用硝酸银进行亲 核取代获得带有 NO 供体基团的白杨素衍生物. 药理研 究表明, 该化合物不仅具有促进细胞增殖的活性, 而且 具有明显的促进血管再生的作用.

2010 年, Dong 等 ${ }^{[29]}$ 合成了带有 NO 供体基团的查尔 酮衍生物，查尔酮在碳酸钾存在的丙酮溶剂中和 1,2-二 溴乙烷发生亲核取代反应生成醚键，从而使 NO 供体基 团与查尔酮连接起来. 应用此法合成 NO 供体化合物, 不仅操作简单且适用于大多数带有酚羟基的天然产物. 应用此方法还可以在多位点同时引入多个 NO 供体基 才. 药理研究表明该化合物与原药相比具有更好的血管 舒张效应，有希望成为新的血管舒张剂.

2010 年, Zou 等 ${ }^{[30]}$ 合成了系列带有 NO 供体基团的 白杨素衍生物. 用二溴代烷在 7 位羟基进行亲核取代反 应，再用硝酸银取代获得目标产物. 药理实验表明该法 合成的化合物其 NO 释放速率比带有羰基的产物要慢, 但其醛糖还原酶抑制性能减弱.

2012 年, Bai 等 ${ }^{[31]}$ 合成了带有 $\mathrm{NO}$ 供体基团的异色 满衍生物. 该合成路线是将异色满的甲氧基取代物进行 选择性去甲氧基反应，再与二溴代烷进行亲核取代反应 后与硝酸银发生亲核取代反应获得带有 NO 供体基团的 异色满衍生物. 药理研究表明, 与卡托普利相比该化合 物具有更好的降压效果.

2013 年, Wang 等 ${ }^{[32]}$ 合成了带有 NO 供体基团的木 犀草素衍生物. NO 的引入增强了原药的醛糖还原酶抑 制活性, 为开发预防和治疗糖尿病并发症药物提供了参 考. Wang 等设计了 3 条合成途径.

第一种途径是先将木犀草素用二溴乙烷处理，用二 溴代烷进行亲核取代后与硝酸银发生亲核取代反应获 得带有 NO 供体基团的木犀草素衍生物. 此类产物在三 条路线得到的产物中醛糖还原酶抑制活性最强.

第二条合成途径是将木犀草素先用二氯二苯基甲烷 处理，将邻二酚羟基保护起来，然后进行二溴代烷的亲 核取代反应，再与硝酸银发生亲核取代反应，最后再将 保护基团去除. 获得带有邻二酚羟基的 NO 供体化合物.

第三条合成途径是将木犀草素用二溴乙烷与邻二 酚羟基反应成环，再用溴乙酸乙酯进行亲核取代，用碱 进行水解处理得到带羧基的产物, 再用二溴代烷进行亲 核取代，然后与硝酸银发生亲核取代反应获得酯键连接 的 NO 供体化合物. 该方法的优点在于引入了酯键，获 得 NO 供体前体药物. 使母体药物与 NO 供体基团在体 内水解后可分别发挥药理作用.

2010 年, Zou 等 ${ }^{[30]}$ 合成了系列带有 NO 供体基团的 白杨素衍生物. 先用溴乙酸乙酯进行亲核取代, 用碱水 
解成酸后与溴代烃发生酯化反应, 最后用硝酸银取代获 得具有 NO 释放能力的白杨素衍生物(Scheme 8). 该方 法运用前体药物理念, 在母体结构与 NO 供体基团之间 引入酯键连接, 使药物进入体内水解后发挥协同作用. 药理研究表明, 该化合物具有更好的降糖作用.

2009 年, Bhandari 等 ${ }^{[33]}$ 合成了一系列具有 NO 释放 功能的降压药. 与大多数有机硝酸酯类化合物的合成一 样, Bhandari 等先合成了具有降血压作用的化合物骨架, 然后用 $\mathrm{AgNO}_{3}$ 进行亲核取代反应获得有机硝酸酯类化 合物(Scheme 9). 药理研究表明, 该化合物不仅具有原 药的降压作用, 而且还具有抗心律失常作用.

同年, Bhandari 等 ${ }^{[34]}$ 合成了具有 NO 供体基团的非 甾体类抗炎药, 该反应是以丙二酸二乙酯为原料经过系 列反应合成溴代药物母体, 再用硝酸银进行亲核取代反 应获得具有 NO 供体基团的非甾体类抗炎药. 在药理活 性方面发现其具有很强的抗炎作用, 同时病理组织研究
发现该化合物具有强抗溃疡作用和粘膜保护作用。这种 合成途径是将来合成没有副作用的非甾体类抗炎药的 重要手段.

2012 年, Mourad 等 ${ }^{[35]}$ 合成了兼具抗癌活性和 NO 释 放能力的查尔酮类化合物. 该路线先通过羟醛缩合反应 获得查尔酮类化合物骨架，然后用溴乙酰溴进行亲核取 代，最后用硝酸银进行亲核取代反应获得目标化合物 (Scheme 10). 药理研究表明, 该化合物具有明显的抗癌 活性，而且对结肠癌和黑素瘤具有一定的选择性.

2012 年, Abuo-Rahma 等 ${ }^{[18]}$ 合成了一系列新的带有 $\mathrm{NO}$ 供体基团的查尔酮衍生物. 该方法先进行亲核取代 反应，再用硝酸银进行亲核取代反应获得 NO 供体化合 物(Scheme 11). NO 释放研究表明该类 NO 供体化合物具 有缓释效果. 药理研究表明, 该化合物与原药相比具有 更好的抗炎活性，且明显降低了原药的副作用，其致胃 肠道溃疡作用明显降低.<smiles>CCOCOc1cc(O)c2c(c1)OC(c1ccccc1)CC2=O</smiles>

图式 8 带有 NO 供体的白杨素衍生物的合成路线 1

Scheme 8 Synthetic routes 1 of chrysin derivatives bearing NO donor

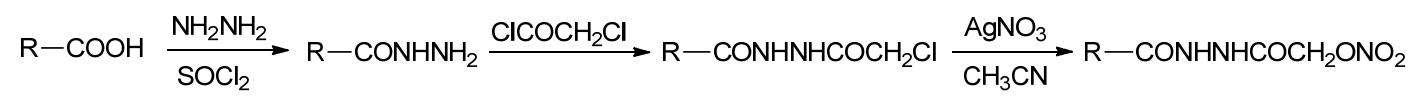

图式 9 羧基连接 NO 供体化合物的合成路线

Scheme 9 Synthetic routes of antihypertensive agents bearing NO donor<smiles>N#Cc1cc(/C=C/C(=O)c2ccc(NC(=O)COO[N+](=O)[O-])cc2)ccc1Cl</smiles>

图式 10 带有 $\mathrm{NO}$ 供体的查尔酮衍生物的合成路线

Scheme 10 Synthetic routes of chalcone derivatives bearing NO donor

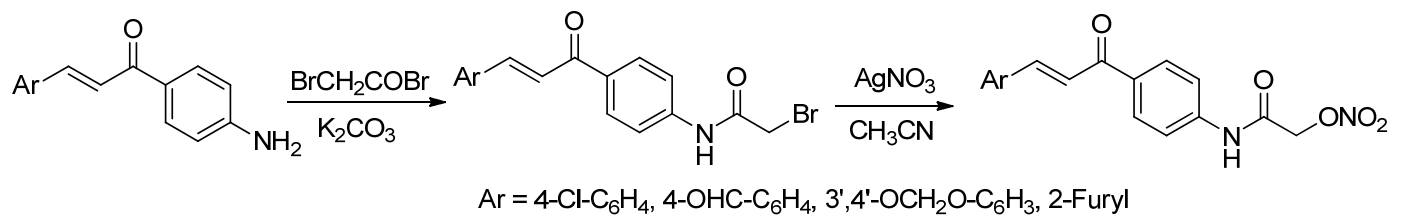

图式 11 带有 $\mathrm{NO}$ 供体的查尔酮衍生物的合成路线

Scheme 11 Synthetic routes of chalcone derivatives bearing NO donor 
Abuo-Rahma 等 ${ }^{[18]}$ 还合成了一组甲基化的 NO 供体 查尔酮衍生物(Scheme 12). 研究表明, 带有甲基的 NO 供体化合物的 NO 释放速率和持续时间都不如没有甲基 取代的 NO 供体化合物. 认为是由空间效应导致.

2016 年, Fang 等 ${ }^{[36]}$ 制备了一种齐墩果酸-NO 供体铂(II)三杂交分子. 该方法先与溴代酰氯进行亲核取代 反应，再用硝酸银进行亲核取代反应得到 NO 供体化合 物, 最后与铂进行连接获得齐墩果酸-NO 供体-铂(II)三 杂交分子. 药理研究表明, 该分子有对肝癌细胞的靶向 细胞毒性，具有协同作用模式和良好的安全性.

2009 年, Zhang 等 ${ }^{[37]}$ 合成了系列带有 NO 供体基团 的抗癌药物厄贝沙坦类似物. 该反应先用二溴代烷进行 亲核取代，接着用硝酸银进行亲核取代反应而获得带有 NO 供体基团的厄贝沙坦类似物(Scheme 13). 药理研究
表明该化合物具有较强的细胞毒性, 且 NO 供体部分对 抗癌活性具有增强作用.

2012 年, Tamboli 等 ${ }^{[38]}$ 在降糖药甲苯磺丁嫝类似物 的结构上添加 $\mathrm{NO}$ 供体获得了具有血管舒张活性和抗血 栓能力的新化合物. 该路线利用丙烯基的双键，一次添 加两个 NO 供体基团(Scheme 14). 此法不仅增加了 NO 的释放还节省了反应过程. 药理研究表明, 该化合物具 有促胰岛素释放效应、血管舒张效应和抗聚活性.

2013 年, Kutty 等 ${ }^{[39]}$ 合成了带有 NO 供体基团的卤代 呋喃酮衍生物. 卤代呋喃酮类化合物是一种从海洋生物 红藻中产生的天然抗菌剂. 该方法是在烷烃链上用 $N$ 溴代丁二酰亚胺(NBS)来进行溴化，再用硝酸银进行亲 核取代反应得到带有 NO 供体的目标化合物(Scheme 15). 药理研究表明, NO 的引入增强了其抗菌活性.

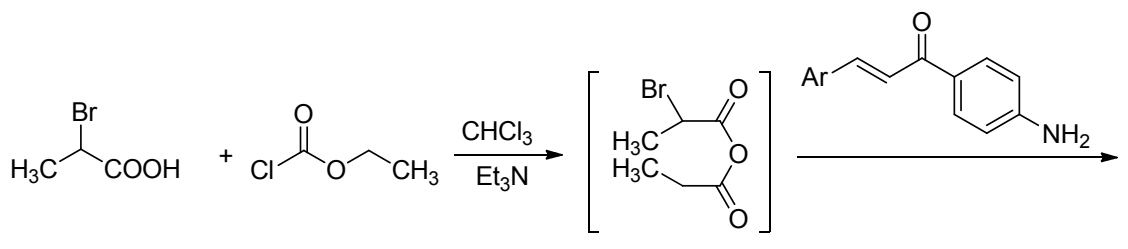<smiles>CC(Br)C(=O)Nc1ccc(C(=O)C(C)C(C)C(=O)O)cc1</smiles>

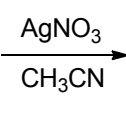<smiles>CC(O[N+](=O)[O-])C(=O)Nc1ccc(C(=O)C(C)C([Al])C(C)[AlH2])cc1</smiles>

Ar: 4- $\mathrm{ClC}_{6} \mathrm{H}_{4}, 4-\mathrm{OHCC}_{6} \mathrm{H}_{4}, 3^{\prime}, 4^{\prime}-\mathrm{OCH}_{2} \mathrm{OC}_{6} \mathrm{H}_{4}, 2$-Furyl

图式 12 甲基化的 $\mathrm{NO}$ 供体查尔酮衍生物的合成路线

Scheme 12 Synthetic routes of methylated chalcone derivatives bearing NO donor

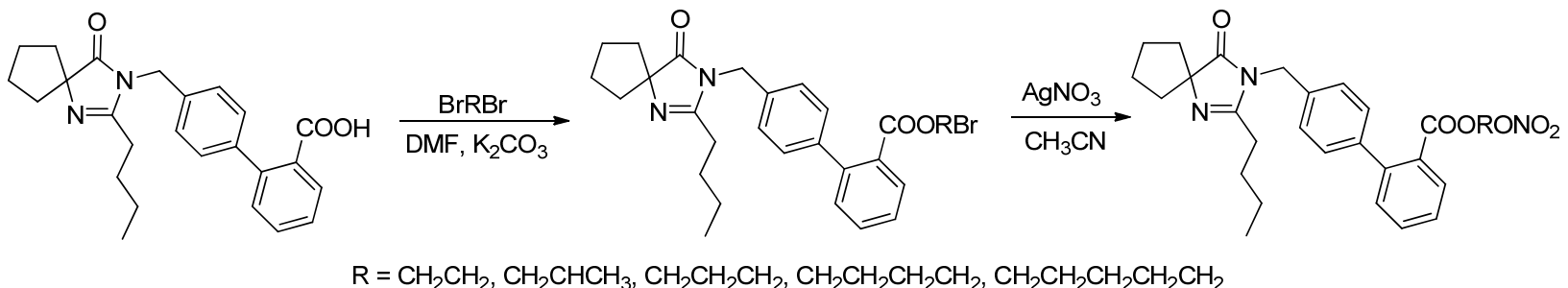

图式 13 带有 NO 供体的厄贝沙坦衍生物的合成路线

Scheme 13 Synthetic routes of irbesartan derivatives bearing NO donor<smiles>C=CCOc1ccc(S(N)(=O)=O)cc1</smiles>

图式 14 带有 NO 供体的甲苯磺丁嫝类似物的合成路线

Scheme 14 Synthetic routes of tolbutamide analogue bearing NO donor 


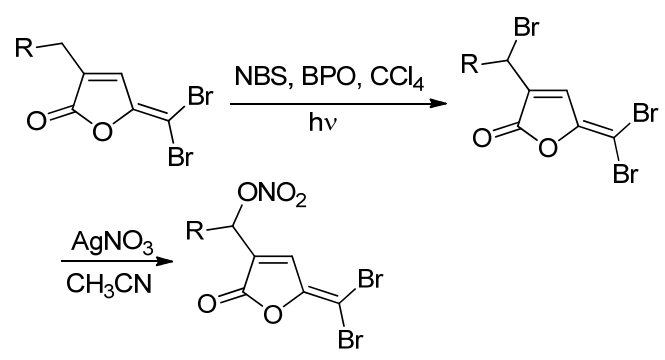

图式 15 带有 NO 供体的卤代呋喃酮衍生物的合成路线 Scheme 15 Synthetic routes of fimbrolide derivatives bearing $\mathrm{NO}$ donor

2015 年, Huang 等 ${ }^{[00]}$ 合成了带有 NO 供体基团的抗 青光眼药物布林佐胺衍生物. 该方法先用三溴化硼进行 脱甲基反应, 用氢溴酸水溶液进行亲核取代, 再与硝酸 银发生亲核取代反应获得带有 $\mathrm{NO}$ 供体基团的布林佐胺 衍生物(Scheme 16). 药理研究表明, 该化合物与原药布 林佐胺相比具有更好降低眼内压作用.
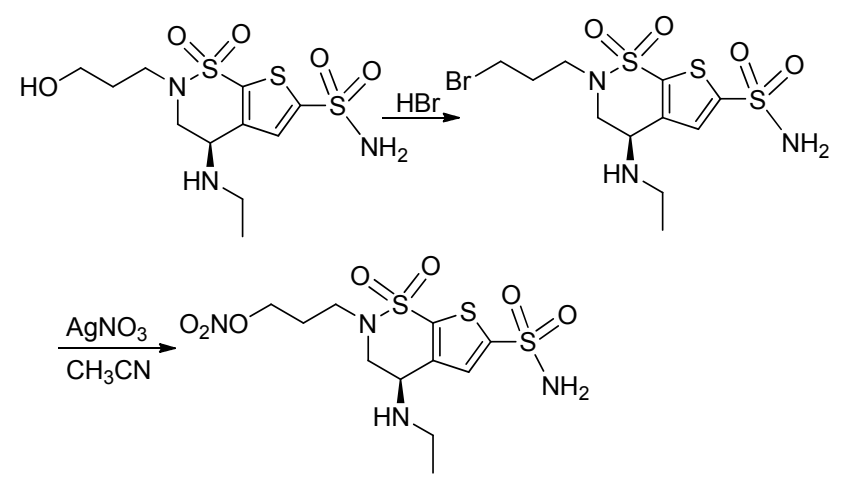

图式 16 带有 $\mathrm{NO}$ 供体的布林佐胺衍生物的合成路线 Scheme 16 Synthetic routes of brinzolamide derivatives bearing $\mathrm{NO}$ donor

\subsection{2 羧酸的 $\alpha-\mathrm{H}$ 取代}

用浓硫酸和浓硝酸对溴代醇进行硝化得到溴代的 NO 供体基团(Eq. 5), 再与母体化合物的羧基发生取代 反应即可将母体化合物与 $\mathrm{NO}$ 供体基团连接(Eq. 6).

$$
\begin{aligned}
& \mathrm{Br} \sim \mathrm{OH} \stackrel{\mathrm{H}_{2} \mathrm{SO}_{4}, \mathrm{HNO}_{3}}{\underset{\mathrm{CH}_{2} \mathrm{Cl}_{2}}{\longrightarrow}} \mathrm{Br} \frown \mathrm{ONO}_{2} \\
& \mathrm{R}-\mathrm{COOH} \stackrel{\mathrm{Br} \bumpeq \mathrm{ONO}_{2}}{\longrightarrow} \mathrm{R}-\mathrm{COO} \bumpeq \mathrm{ONO}_{2}
\end{aligned}
$$

2008 年, Abdellatif 等 ${ }^{[41]}$ 合成了带有 NO 供体基团的 丙烯酸衍生物. 该方法是直接用 2-溴乙基硝酸酯将丙烯 酸衍生物的 $\alpha$ 位 $\mathrm{H}$ 取代获得带有 $\mathrm{NO}$ 供体基团的丙烯酸 衍生物. 药理研究表明, 该化合物不仅具有比原药更强 的抗炎活性，而且其胃肠道不良反应明显降低.

2012 年, Kaur 等 ${ }^{[42]}$ 合成了带有 NO 供体基团的抗 II 型糖尿病药物美各里替尼衍生物. 该合成路线先合成了
药物美各里替尼, 再与带有 $\mathrm{NO}$ 供体基团的澳代物发生 羧基的 $\alpha$ 位 $\mathrm{H}$ 取代反应获得目标化合物. 药理研究表明, 与原化合物相比该 $\mathrm{NO}$ 供体化合物具有更好的降血糖效 果. 可以用来治疗 II 型糖尿病及其引起的血管并发症.

2015 年, $\mathrm{Xu}$ 等 ${ }^{[43]}$ 合成了带有 $\mathrm{NO}$ 供体基团的乙丙昔 罗衍生物. 该合成路线是将溴代醇进行硝化处理, 再将 $\mathrm{NO}$ 供体基团通过羧基的 $\alpha$ 位 $\mathrm{H}$ 取代反应连接到乙丙昔 罗骨架上获得 NO 供体化合物. 该化合物具有较好的 $\mathrm{NO}$ 释放能力. $\mathrm{Xu}$ 等还合成了以溴丙醇为起始原料的产 物, $\mathrm{NO}$ 释放研究表明其释放速率和缓释性能都会降低.

2016 年, Gazzano 等 ${ }^{[4]}$ 合成了带有 NO 供体基团的 多柔比星衍生物. 该路线是将 NO 供体基团通过羧基的 $\alpha$ 位 $\mathrm{H}$ 取代反应连接到多柔比星骨架上获得 NO 供体化 合物. 药理研究表明, 连接两个 NO 供体基团的化合物 比连接一个 NO 供体的化合物能够释放更多的 NO. 同 时该类化合物还具有一定选择性，只在对多柔比星敏感 的肿瘤细胞中增加 NO, 从而增强细胞毒性.

\subsection{3 胺的取代}

用发烟硝酸对溴代醇进行处理发生硝化反应，得到 溴代硝酸酯化合物. 溴代硝酸酯与带有氨基的药物母体 发生取代反应即可得到 NO 供体化合物(Scheme 17).

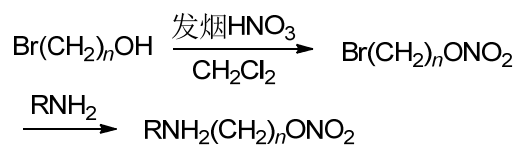

图式 17 胺取代合成 NO 供体化合物

Scheme 17 Synthesis of NO donor compounds by substitution of amines

2008 年, Fang 等 ${ }^{[45]}$ 合成了带有 NO 供体基团的抗老 年痴呆药物他克林衍生物. 该方法是先合成带有氨基基 团的他克林衍生物，再将用发烟硝酸硝化后的溴代烷通 过取代反应连接上去. 药理研究表明, 新合成的 NO 供 体化合物与他克林相比具有较低的肝毒性.

2010 年, Chowdhury 等 ${ }^{[46]}$ 合成了带有 NO 供体基团 的塞来昔布类似物. 此反应是先将塞来昔布的吡啶环进 行还原, 然后再与 NO 供体基团通过取代反应获得具有 双重效应的化合物. 药理研究发现其不仅能释放 NO 而 且具有和阿司匹林相当的抗炎效果.

2011 年, Bertinaria 等 ${ }^{[47]}$ 合成了包含 NO 供体基团的 治疗脑型疮疾的药物阿莫地喹类似物. 该反应是先将中 间体进行硝化，再用茮基氯进行亲核取代反应获得带有 $\mathrm{NO}$ 供体基团的化合物. 药理研究表明, 该化合物与原 药相比具有更快更好的杀灭㾏原虫的能力.

2016 年, Zhao 等 ${ }^{[48]}$ 将硝酸酯 NO 供体连接到钌(II) 芳烃配合物. 药理研究表明, 它们的组合对癌细胞具有 
阳性协同效应. 与游离配体或非官能化复合物相比, 具 有 $\mathrm{NO}$ 释放能力的 4-硝基氧基甲基-吡啶配体的 $\mathrm{Ru}(\mathrm{II})$ 芳烃络合物显示出对非小细胞肺癌细胞系 A549 增加的 细胞毒性.

综上所述, 通过酯键连接的 NO 供体药物可以在体 内水解后发挥相连的两部分的各自功能. Zou 等 ${ }^{[30]}$ 采用 的合成方案具有产率高、污染少、易于操作的优点. 其 反应过程是先与药物母核上的羟基成酯，再用碱处理成 酸, 得到的产物经溴代烷取代后进行硝化. 此反应产率 高且属于绿色化学, 是有机硝酸酯类 NO 供体药物合成 的较好方案. 另外, Tamboli 等 ${ }^{[38]}$ 采用引入溴丙烯基的合 成方案实现了一次性引入两个有机硝酸酯类 NO 供体基 团的可能, 对增强 NO 的体内释放有很好的效果. 但此 类药物也有缺点, 有机硝酸酯类药物的耐受性 ${ }^{[49]}$ 大大 限制了其在临床的应用.

\section{2 呋咱氮氧化物类 NO 供体的合成}

呋咱氮氧化物类化合物对酸碱均较稳定, 环上的 3、4 位可连接相同或不同的取代基, 也可以与其他环骈 合而形成不同的衍生物. 这类化合物在进入体内后会在 統基化合物作用下释放 NO. 如 Scheme 18 所示, 呋咱氮 氧化物类化合物在体内先经放基化合物对其 3 或 4 位进 行亲核加成获得加成产物(1 或 4), 再经开环反应形成亚 硝基衍生物(2 或 5), 并进一步生成另一亚硝基衍生物(3 或 6)和亚硝酰基阴离子 $\left(\mathrm{NO}^{-}\right)$. 其中亚硝基衍生物可作 为终产物排出体外, 也可继续水解或硫解, 还可与硫醇 作用, 生成 $S$-亚硝基硫醇, 然后裂解生成 NO. 而亚硝酰
基阴离子经氧化形成 $\mathrm{NO}$ 释放 ${ }^{[50]}$.

经典的有机硝酸酯类 NO 供体化合物需在游离放基 作用下才能释放 NO, 而呋咱氮氧化物类 NO 供体化合 物在游离放基和颈基阴离子作用下均可释放 NO. 因为 体内游离放基分布很广, 所以呋咱氮氧化物类 NO 供体 化合物可在多种组织或器官中通过非酶催化途径释放 NO 而产生一定的生物活性. 不诱导产生耐受性也是呋 咱氮氧化物类 NO 供体化合物的优点, 典型的呋咱氮氧 化物类化合物包括苯基取代、苯磺酰基取代和甲基取代 的呋咱氮氧化物等.

\section{1 酯化反应}

以肉桂醇为原料, 在酸性条件下加入亚硝酸钠盐反 应得呋咱氮氧化物类 NO 供体基团 3-羟甲基-4-苯基1,2,5-噁二唑-2-氧化物. 此化合物与带有羧基的药物分 子进行酯化反应得 NO 供体化合物(Scheme 19).

2007 年, López 等 ${ }^{[20]}$ 合成了带有 NO 供体基团的 $\alpha$-生 育酚类似物. 该路线采用成酯反应将 NO 供体基团通过 酯键连接到水溶性维生素 $\mathrm{E}$ 结构上(Eq. 7). 该方法合成 的化合物在体内经酶水解后的产物可各自产生相应的 活性. 药理研究表明该化合物具有 NO 释放效应，血管 舒张效应和哺乳动物细胞毒性效应.

2008 年, Chen 等 ${ }^{[51]}$ 合成了带有 $\mathrm{NO}$ 供体基团的齐墩果 酸衍生物. 该合成路线是将齐墩果酸与丁二酸酐进行成 酯反应，得到的产物再与带有羟基的 NO 供体基团进行 酯化反应, 获得了带有 NO 供体基团的齐墩果酸衍生物. 药理研究表明, 该化合物在体内能够在肝癌细胞中高水 平释放, 而且对肝癌细胞具有高度选择性的细胞毒性.

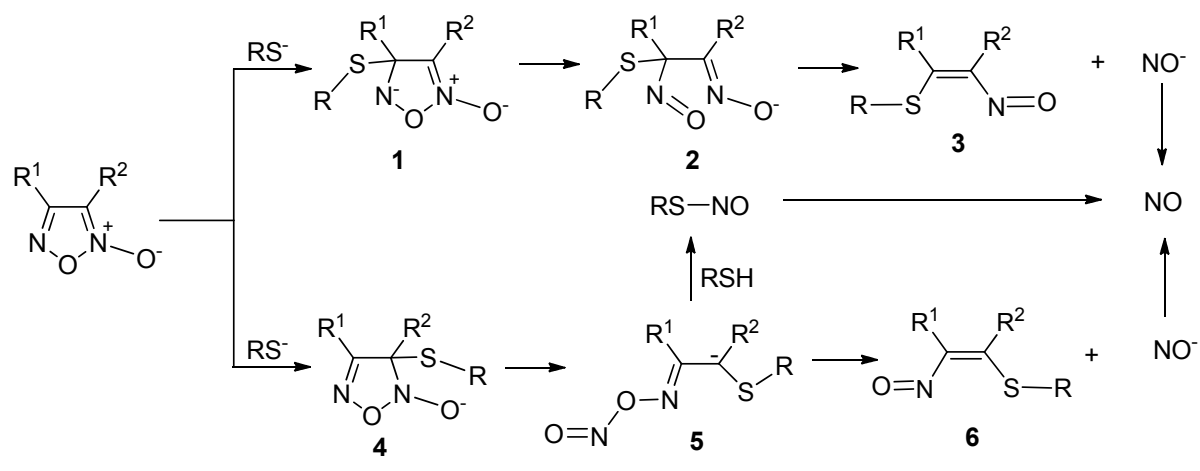

图式 18 呋咱氮氧化物类化合物体内释放 NO 的机制

Scheme 18 Mechanism of releasing NO from furoxans in vivo

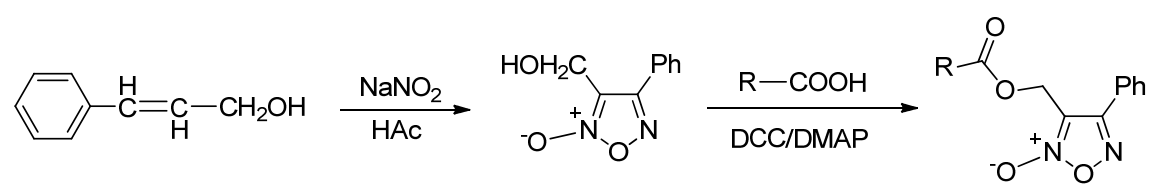

图式 19 酯化反应制备 NO 供体化合物

Scheme 19 Synthesis of NO donor compound by esterification 
<smiles>Cc1c(C)c2c(c(C)c1O)CCC(C)(C(=O)O)O2</smiles><smiles>Cc1c(C)c2c(c(C)c1O)CCC(C)(C(=O)OCCCOc1no[n+]([O-])c1S(=O)(=O)c1ccccc1)O2</smiles>

2009 年, Peng 等 ${ }^{[28]}$ 合成了带有 NO 供体基团的白杨 素衍生物. 该路线用白杨素衍生物对溴乙酸乙酯进行亲 核取代反应, 碱处理得到带有羧基的中间产物, 再与以 肉桂醇为原料合成的 NO 供体基团进行成酯反应获得带 有 NO 供体基团的白杨素衍生物. 鸡胚线毛尿囊膜试验 表明该化合物显著的促进血管生成作用.

2009 年, Zhang 等 ${ }^{[37]}$ 合成了系列带有 NO 供体基团 的厄贝沙坦衍生物. 该方法是先以苯硫酚为原料合成带 有不同取代基的 NO 供体基团, 再与厄贝沙坦进行偶合 获得带有 NO 供体基团的厄贝沙坦衍生物. 药理研究表 明该化合物有较好的细胞毒性活性, 引入 NO 供体基团 对抗癌活性有增强作用.

2010 年, Ling 等 ${ }^{[52]}$ 合成了带有 NO 供体基团的法尼 基硫代水杨酸(FTA)衍生物. 该路线以苯硫酚为原料合 成 NO 供体基团, 再与 $L$-丙氨酸衍生物结合, 最后与苯
甲酸衍生物进行偶合获得 NO 供体化合物. 药理研究表 明，该化合物不仅在体内具有较高水平的 NO 释放性能， 而且还具有很好的抗癌活性.

2010 年, Zou 等 ${ }^{[30]}$ 合成了系列带有呋咱氮氧化物类 NO 供体基团的白杨素衍生物. 药理研究表明, 该化合 物对醛糖还原酶有抑制作用且能够释放 NO. 该化合物 对预防和治疗糖尿病及其引发的血管并发症有一定效 果. Zou 等设计了两条合成路线.

路线 1 是先将白杨素的 7 位羟基用溴乙酸乙酯进行 取代, 然后用碱处理得到羧酸产物, 再跟与对差基苯甲 醇相连的 NO 供体基团进行成酯反应，最后再用酸酐进 行缩合反应获得带有 NO 供体基团的白杨素衍生物 (Scheme 20).

路线 2 采用的方法是用白杨素的羧酸衍生物与带有 差基的 NO 供体基团直接通过酯键连接起来, 然后再用 酸酐进行缩合反应获得带有 NO 供体基团的白杨素衍生 物(Eq. 8). NO 释放研究表明, NO 释放均比较缓慢, 但路 线 2 得到的化合物释放更慢.

2011 年, Shi 等 ${ }^{[53]}$ 合成了系列带有 NO 供体基团的 核苷衍生物. 该方法利用成酯反应将 NO 供体基团与核 苷衍生物连接. 该化合物在体内经水解后各部分可发挥 各自活性. 经药理研究表明，带有 NO 供体基团的核苷 衍生物比药物阿昔洛韦和司他夫定有更好的抗病毒活 性(Scheme 21).

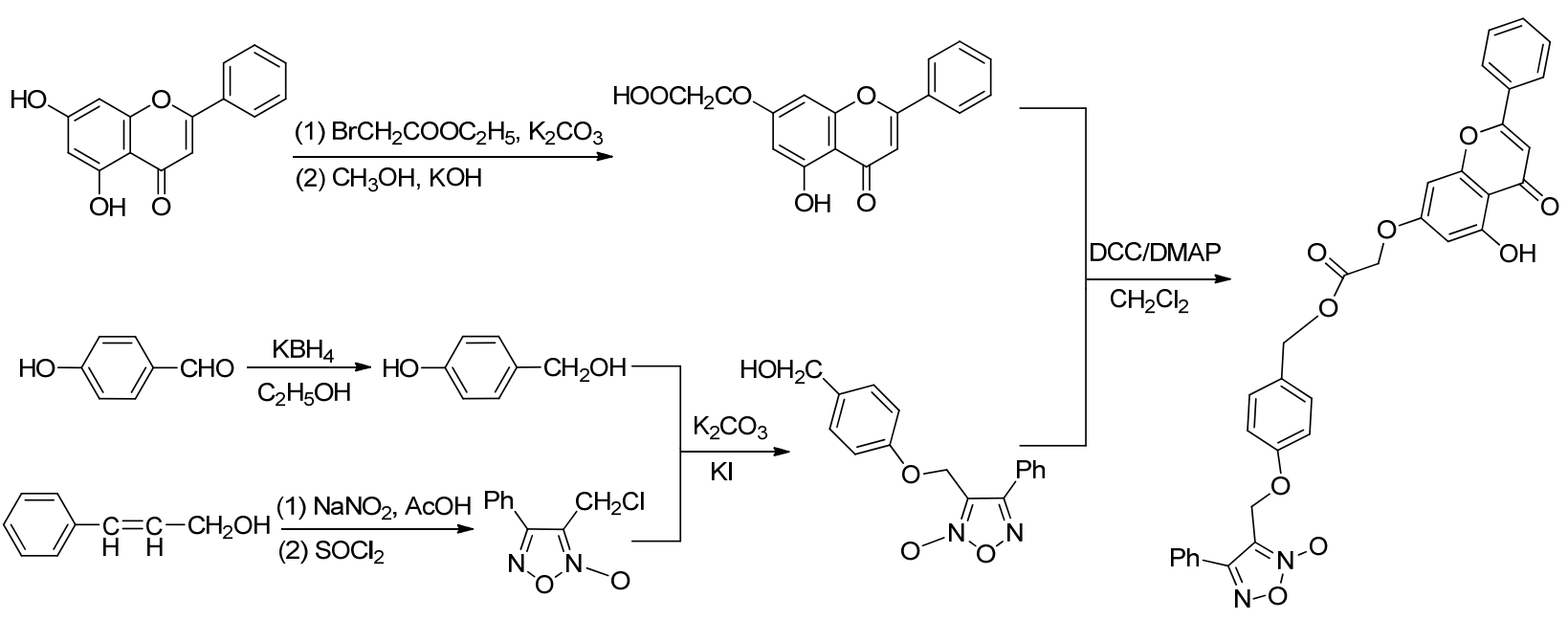

图式 20 带有 NO 供体的白杨素衍生物的合成路线

Scheme 20 Synthetic route of chrysin derivatives bearing NO donor<smiles>O=C(CO)Oc1cc(O)c2c(=O)cc(-c3ccccc3)oc2c1</smiles>

DCC/DMAP, $\mathrm{CH}_{2} \mathrm{Cl}_{2}$<smiles></smiles><smiles>O=C(COc1cc(O)c2c(=O)cc(-c3ccccc3)oc2c1)OCc1c(-c2ccccc2)no[n+]1[O-]</smiles> 


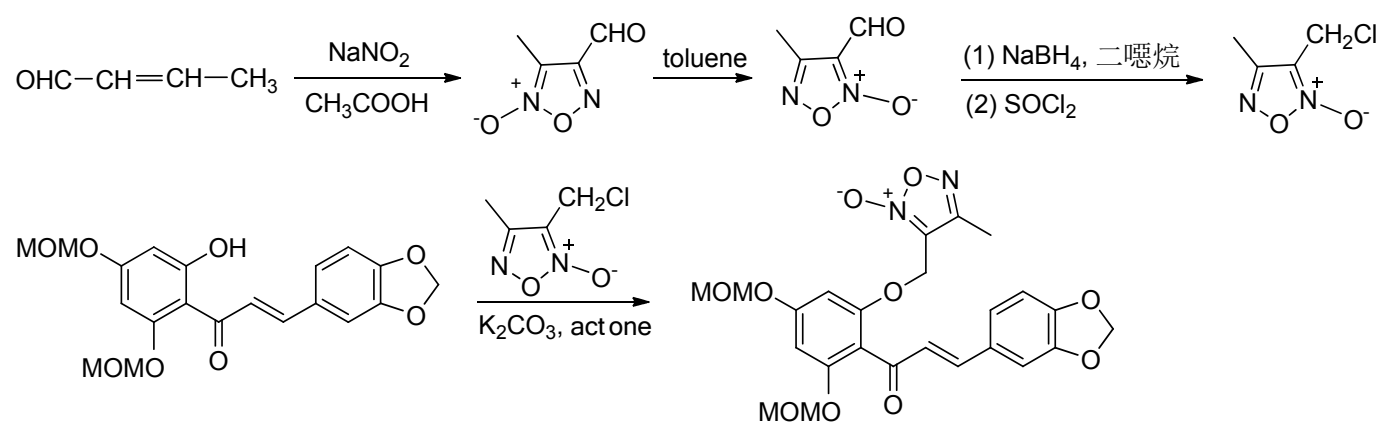

图式 21 带有 NO 供体的查尔酮衍生物的合成路线

Scheme 21 Synthetic routes of chalcone derivative bearing NO donor

2012 年, Mourad 等 ${ }^{[35]}$ 合成了系列带有 NO 供体基团 的查尔酮衍生物。该方法是先合成查尔酮化合物骨架， 再与呋咱氮氧化物类 $\mathrm{NO}$ 供体基团经亲核取代反应连接 起来. 药理研究表明, 该化合物对不同的癌细胞都具有 细胞毒性活性, 但其活性不如有机硝酸酯类 NO 供体药 物显著.

2012 年, Abuo-Rahma 等 ${ }^{[18]}$ 合成了带有 NO 供体基 团的查尔酮衍生物. 该路线是先用 4 -氯苯甲醛和对氨基 苯乙酮经过羟醛缩合反应合成查尔酮化合物骨架，再与 溴乙酰溴进行偶联, 得到的产物再与 $\mathrm{NO}$ 供体基团结合 获得 NO 供体化合物. 药理研究表明, 该化合物与查尔 酮相比其抗炎活性增强，而且该化合物还降低了查尔酮 的致溃疡副作用.

2014 年, Tang 等 ${ }^{[54]}$ 合成了带有 NO 供体基团的鸦胆 子苦醇衍生物. 药理研究表明, 该化合物对吸烟引起的 慢性肺炎具有抑制作用. 该路线利用加入的丁二酸䣶作 为 $\mathrm{NO}$ 供体基团与鸦胆子苦醇的连接基团, 利用酯键连 接可使化合物在体内水解后的产物发挥各自效应. 药理 研究表明, 该化合物具有较好的抗炎作用, 且由于 NO 供体基团的引入, 其毒性也大幅降低.

\section{2 取代反应}

2010 年, Dong 等 ${ }^{[29]}$ 合成了系列带有 NO 供体基团的 查尔酮衍生物. 该方法是将 2-丁烯醛经亚硝酸钠处理后 成氧化呋咱环, 在沸腾的甲苯中加热 $98 \mathrm{~h}$ 后转化成其同 分异构体, 再进行还原和卤代, 然后与查尔酮化合物进 行偶联, 最后进行脱二甲醚反应得到带有 NO 供体基团 的查尔酮衍生物(Scheme 21). 该方法特点在于用甲苯 对 NO 供体进行了同分异构转化. 药理活性研究表明, 该化合物由于 $\mathrm{NO}$ 供体基团的引入使其血管舒张性能比 原化合物更强, 有希望成为新的血管舒张剂.

2011 年, Bertinaria 等 ${ }^{[47]}$ 合成了系列带有 NO 供体基 团的抗脑型疮疾药物阿莫地喹类似物. 此方法利用苯磺 酰基在亲核取代反应中易离去的特性使 $\mathrm{NO}$ 供体基团与
母体药物更易结合. 该方法是将呋咱氮氧化物类 NO 供 体基团与乙基差乙胺进行偶联, 得到的中间产物再与阿 莫地喹类似物进行偶联得到带有 NO 供体基团的抗脑型 症疾药物阿莫地喹类似物. 药理研究表明, 得到的 NO 供体化合物具有阿莫地喹没有的血管舒张活性.

2012 年, Tamboli 等 ${ }^{[38]}$ 合成了一系列带有 NO 供体 基团的降糖药物甲苯磺丁脲的类似物. 该方法是先将 $\mathrm{NO}$ 供体基团和 4-磺酰氨基苯酚偶联, 再与异氧酸丁酯 偶联获得带有 NO 供体的甲苯磺丁腿的类似物(Eq. 9). 药理研究表明, 与呋咱氮氧化物类 NO 供体基团偶合的 产物比与有机硝酸酯类 $\mathrm{NO}$ 供体基团偶合产物的促胰岛 素释放活性更高.

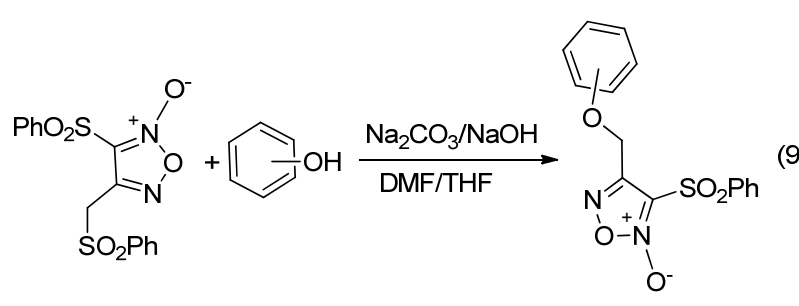

2012 年, Dos Santos 等 ${ }^{[5]}$ 利用酸酐的氨解合成了带 有 NO 供体基团的苯邻二甲酰亚胺衍生物。该反应是用 邻苯二甲酸䣶和呋咱氮氧化物类 NO 供体基团通过酰化 反应得到 NO 供体化合物(Eq. 10). 药理研究表明, 该化 合物具有多因子抗氧化活性，且具有阻止缺血再灌注损 伤的作用。

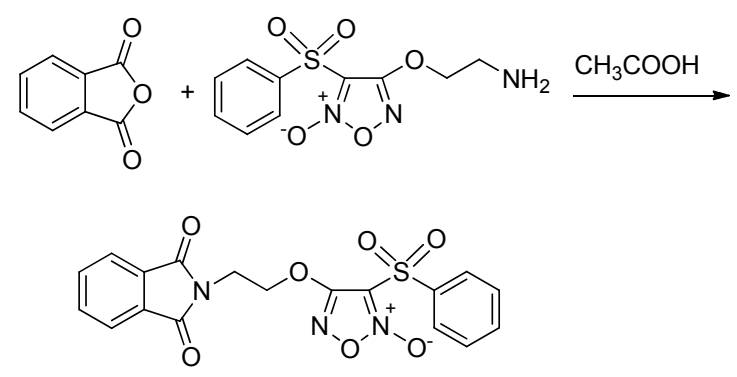


2016 年, Gazzano 等 ${ }^{[44]}$ 合成了带有 NO 供体基团的 多柔比星衍生物. 该路线是将 NO 供体基团通过羧基的 $\alpha$ 位 $\mathrm{H}$ 取代反应连接到多柔比星骨架上获得 NO 供体化 合物(Eq. 11). 药理研究表明, 该化合物的 NO 释放能力 与连接两个硝酸酯 $\mathrm{NO}$ 供体基团的化合物相当.

2015 年, Bertinaria 等 ${ }^{[56]}$ 合成了系列带有 NO 供体基 团的二氢青蒿素衍生物. 该路线是将 NO 供体基团通过 取代反应连接到二氢青蒿素骨架上获得 NO 供体化合物 (Scheme 22). 药理研究表明, 该类化合物在体外和体内 对抗伯氏症原虫实验中显示出较好活性, 而且在大鼠主 动脉条的血管舒张效力中其活性比呋咱氮氧化物类 $\mathrm{NO}$ 供体强.

\section{3 成肽反应}

2011 年, Bian 等 ${ }^{[57]}$ 合成了带有 NO 供体基团的抗白
血病药物他米巴罗汀衍生物. 该方法是先合成了氯取代 的 NO 供体基团，再通过成肽反应将 NO 供体和药物他米 巴罗汀连接(Eq. 12). 药理研究表明, $\mathrm{R}$ 为氢或苠基取代 时, 得到的 NO 供体化合物抗白血病活性要比原药更强. 同时 NO 释放研究表明，带有氨基酸的 NO 供体化合物其 释放率更高, 而且其抗白血病活性也是一致增高的.

2013 年, Borretto 等 ${ }^{[58]}$ 合成了带有 NO 供体基团的 恩替诺特衍生物. 该合成路线是用叔丁基二甲基硅烷基 保护的 5-(差圣甲基)吡啶-2-甲醛由甲胺和嗍氢化钠处理进 行还原性胺化得到中间体，再用 4-溴甲基氧化呋咱甲酰 胺处理得到氧化呋咱中间体，经乙酸处理后与中间体 $\mathbf{8}$ 经成肽反应得到氧化呋咱羧酰胺衍生物 $\mathbf{9}$, 这一产物经 三氟乙酸酐脱水处理后得到相关的腈，随后用甲磺酸将 其去保护，得到目标化合物(Eq. 13).

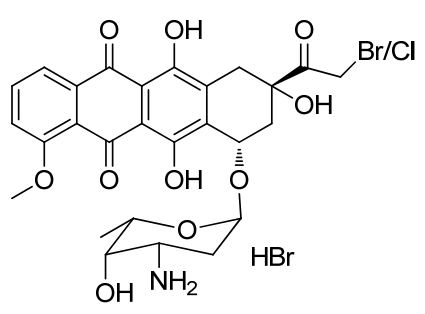<smiles>COn1nc(COc2ccc(C(=O)O)cc2)c(S(=O)(=O)Oc2ccccc2)[n+]1[O-]</smiles><smiles>COc1cccc2c1C(=O)c1c(O)c3c(c(O)c1C2=O)C[C@@](O)(C(=O)COC(=O)c1ccc(OCc2no[n+]([O-])c2S(=O)(=O)c2ccccc2)cc1)C[C@H]3OC1CC(N)C(O)C(O)C1</smiles><smiles>CC1CCC2C(C)C(OCCBr)OC3OC4(C)CCC1C32OO4</smiles>

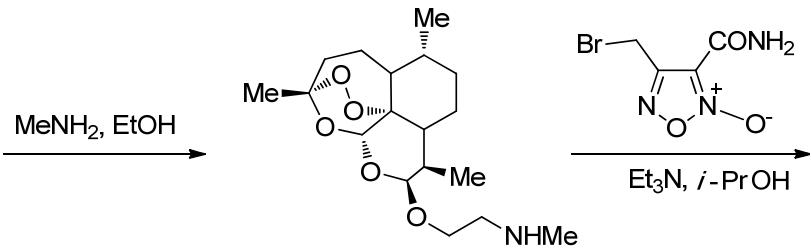

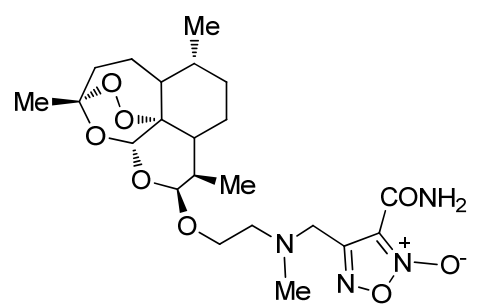

图式 22 带有 NO 供体的二氢青蒿素衍生物的合成路线

Scheme 22 Synthetic routes of dihydroartemisinin derivative bearing NO donor<smiles>[R]C(N)C(=O)OCc1c(-c2ccccc2)no[n+]1[O-]</smiles><smiles>[R]C(NC(=O)c1ccc(C(=O)Nc2ccc3c(c2)C(C)(C)CCC3(C)C)cc1)C(=O)OCc1c(-c2ccccc2)no[n+]1[O-]</smiles><smiles></smiles> 
2013 年, $\mathrm{Lu}$ 等 ${ }^{[59]}$ 合成了带有 $\mathrm{NO}$ 供体基团的羟基肉 桂酸衍生物. 该路线是先合成带有氨基的 NO 供体基团, 与乙酸䣶保护的羟基肉桂酸衍生物通过成肽反应连接, 最后脱去羟基保护基团获得带有 $\mathrm{NO}$ 供体基团的羟基肉 桂酸衍生物(Scheme 23). 药理研究发现, 该化合物具有 比 5-氟尿嘧啶更强的抗癌活性.

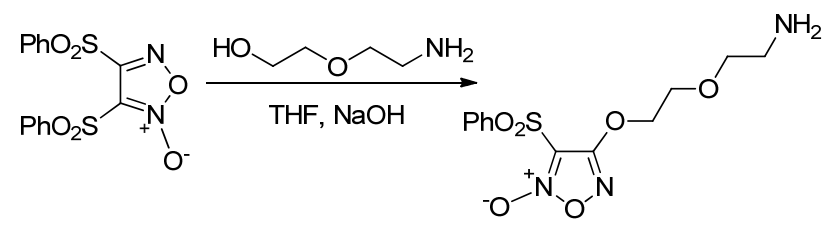<smiles>CCOc1ccc(/C=C/C(=O)OCCNC(=O)/C=C/c2ccc(OC(C)=O)c(OC(C)=O)c2)cc1OC(C)=O</smiles>

图式 23 带有 $\mathrm{NO}$ 供体的羟基肉桂酸衍生物的合成路线 Scheme 23 Synthetic routes of hydroxylcinnamic acid derivative bearing $\mathrm{NO}$ donor

2015 年, $\mathrm{Ai}$ 等 ${ }^{[60]}$ 合成了带有 $\mathrm{NO}$ 供体基团的齐墩果 酸衍生物. 该路线是先将 NO 供体基团与 2-丁炔-1,4-丁 二醇连接，再在碳化二亚胺等作用下形成肽键，最后与 酰氯化的齐墩果酸衍生物偶合获得 NO 供体基团的齐墩 果酸衍生物(Scheme 24). 药理研究表明, 由于 NO 供体 基团的加入使该化合物治疗耐药性结肠癌有较好的疗 效.

2015 年, Duan 等 ${ }^{[61]}$ 合成了系列带有 NO 供体基团的 组蛋白去乙酰化酶抑制剂衍生物. 该路线是以 2-苯硫基 乙酸为原料合成 $\mathrm{NO}$ 供体基团, 再经二羟基化合物取代, 经琼斯试剂氧化成羧酸, 经成肽反应后得到带有 NO 供 体基团的组蛋白去乙酰化酶抑制剂衍生物(Eq. 14). 药 理研究表明, 该化合物具有抗白血病细胞增殖的作用, 其抗癌活性与 $\mathrm{NO}$ 的体内释放有关.

\section{4 醛胺缩合}

2014 年, Massarico Serafim 等 ${ }^{[62]}$ 合成了带有 NO 供 体基团的酰基腙衍生物. 该方法是先用反式肉桂酸合成 呋咱氮氧化物类 NO 供体基团, 再与酰肼类化合物进行 醛胺缩合生成酰腙获得 NO 供体化合物(Scheme 25). 药 理研究表明, 这类化合物具有很强的抗锥虫活性, 与原 药相比具有更高的渗透性和更好的选择性.

2016 年, Lakshman 等 ${ }^{[63]}$ 制备了带有 NO 供体基团的 锌(II)-NSAID 络合物. 该方法是将 NO 供体连接到锌(II) 后再与非甾体类抗炎药形成 NO 供体络合物. 药理研究 表明, 得到的络合物不仅具有较好的抗炎活性而且还
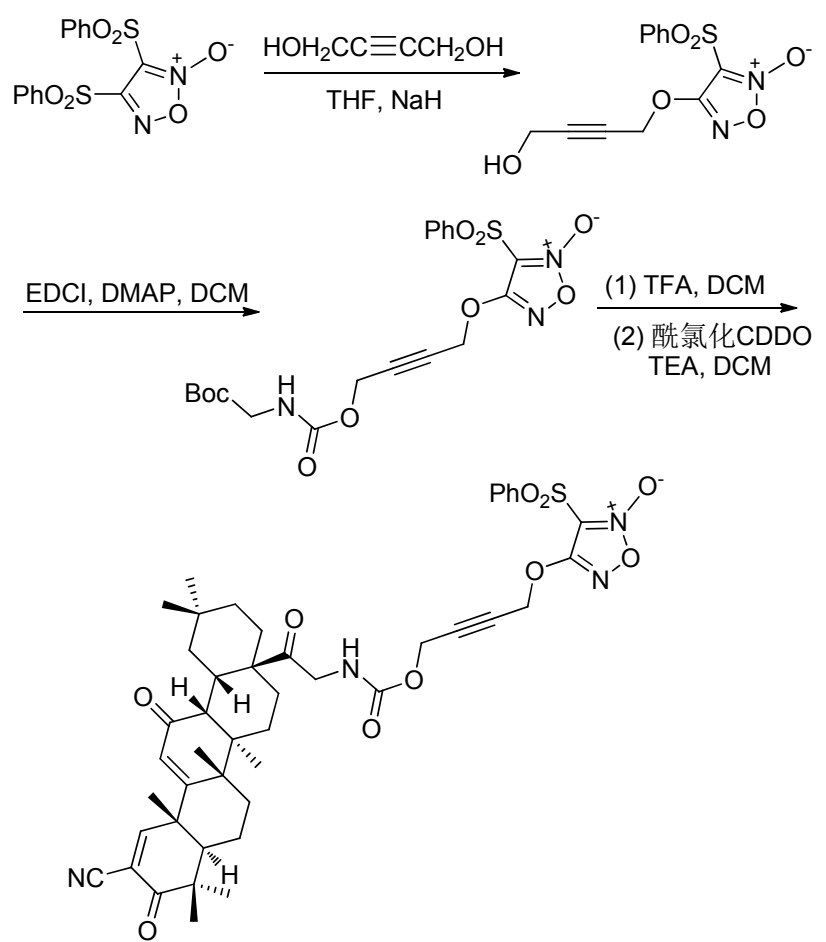

图式 24 带有 NO 供体的齐墩果酸衍生物的合成路线 Scheme 24 Synthetic routes of oleanolic acid derivative bearing NO donor

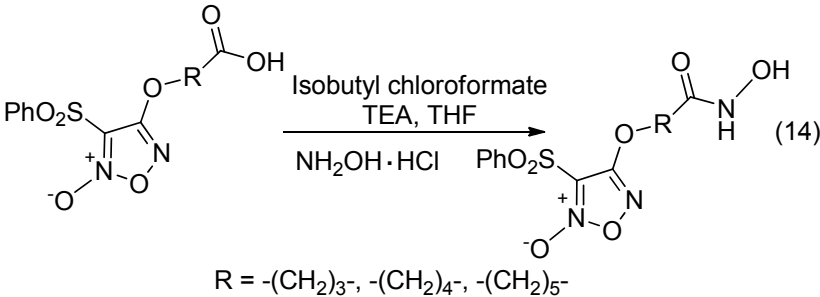

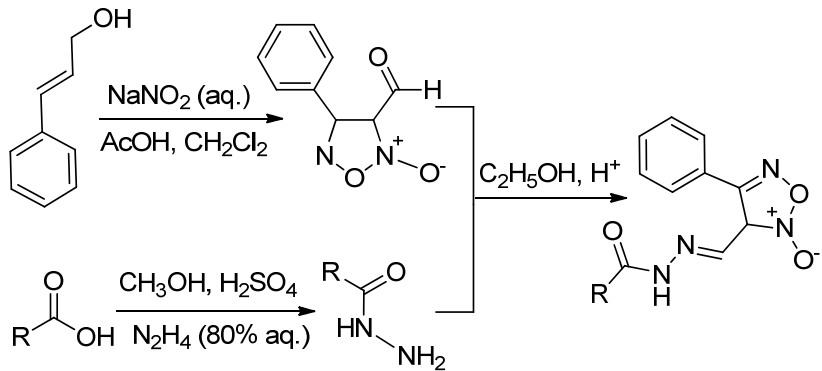<smiles>[X]c1ccc([N+](=O)[O-])c([N+](=O)[O-])c1</smiles>

图式 25 带有 $\mathrm{NO}$ 供体的酰基腙衍生物的合成路线 Scheme 25 Synthetic routes of acylhydrazone derivatives bearing $\mathrm{NO}$ donor

具有 COX-2 选择抑制活性. 该方法是将非选择性或 COX-1 选择性 NSAID 转化为选择性 COX-2 抑制剂的代 
表途径.

综上所述, Peng 等 ${ }^{[28]}$ 采用的合成方法具有产率高、 无污染、操作简单等优点. 其采用的合成路线是先以肉 桂醇为原料合成了带有羟基的呋咱氮氧化物类 $\mathrm{NO}$ 供体 基团, 然后再与带有羧基的查尔酮衍生物进行成酯反应 得到呋咱氮氧化物类 $\mathrm{NO}$ 供体药物. 该合成路线简便安 全, 绿色环保, 是很好的合成 $\mathrm{NO}$ 供体药物的方法.

\section{3 偶氮二醇烯鎓盐类 NO 供体的合成}

偶氮二醇烯鎓盐一般可由哌嗪、吡咯、烷基胺或哌 啶与 $\mathrm{NO}$ 和甲醇钠的甲醇溶液在 $\mathrm{NO}$ 吸附剂(如纳米二氧 化钛)催化下反应生成, 代表药物有 JS-K 和 DETANONOate. 该类化合物的生物半衰期可通过引入取代基 来灵活调节 ${ }^{[64]}, 2$ 个 $\mathrm{N}-\mathrm{O}$ 上烷基化取代可使其半衰期从 几分钟到几小时不等, 且某些与特定基团结合的偶氮二 醇烯鎓盐化合物可在特定部位释放出定量的 NO, 所以 特别适合偶联特定载体制成靶向释放的 NO 供体药物 [65]. 释放时, 在体内经过生物酶的催化形成氧负离子, 氧负离子在体内环境下不稳定, 极易释放出 NO.

\section{$3.1 \mathrm{NO}$ 气体制备}

2008 年, Chakrapani 等 ${ }^{[6]}$ 合成了具有抗癌活性的 JS-K 类似物. 该合成路线是用脯氨醇为原料合成偶氮 二醇烯鈆盐化合物, 再用 2,4-二硝基氟苯和叔丁醇在碳 酸氢钠处理下合成目标化合物 10 (Scheme 26). 药理研 究表明, 该类 NO 供体基团的引入并未对此药物的抗癌 活性产生影响.

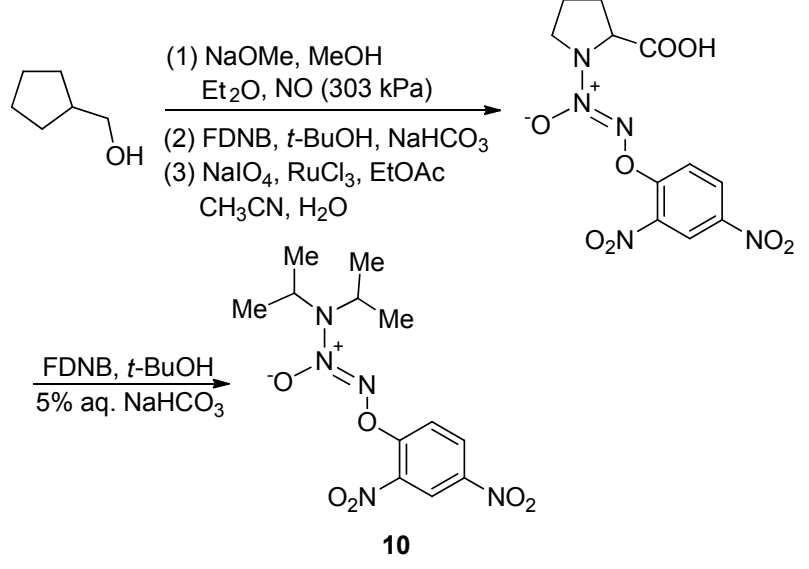

图式 26 JS-K 类似物的合成路线

Scheme 26 Synthetic routes of JS-K analogue

2008 年, Fang 等 ${ }^{[45]}$ 合成了带有 NO 供体基团的抗老 年痴呆药物他克林衍生物. 该合成路线是先用吡咯烷与 $\mathrm{NO}$ 气体反应合成 NO 供体基团, 以邻氨基苯甲酸和环 己酮为原料经过一系列反应合成溴代他克林衍生物, 此 衍生物再与 $\mathrm{NO}$ 供体进行偶合获得带有 $\mathrm{NO}$ 供体基团的
他克林衍生物(Eq. 15). 在肝中毒研究中发现, 该化合物 并不会像药物他克林一样引起严重的肝中毒.<smiles>CC(=O)ON1CCC(Br)C1</smiles><smiles></smiles>

2008 年, Konter 等 ${ }^{[24]}$ 合成了带有 NO 供体基团的酮 康唑衍生物. 该合成路线是将酮康坐化合物经 $\mathrm{NaOH}$ 水 溶液水解后与 $\mathrm{NO}$ 气体在甲醇钠中反应生成带有偶氮二 醇烯鎓盐类 NO 供体基团的酮康唑衍生物(Scheme 27). 药理研究发现该化合物的抗菌活性明显比原药酮康唑 更强，而单独的 NO 供体则没有这种效果.
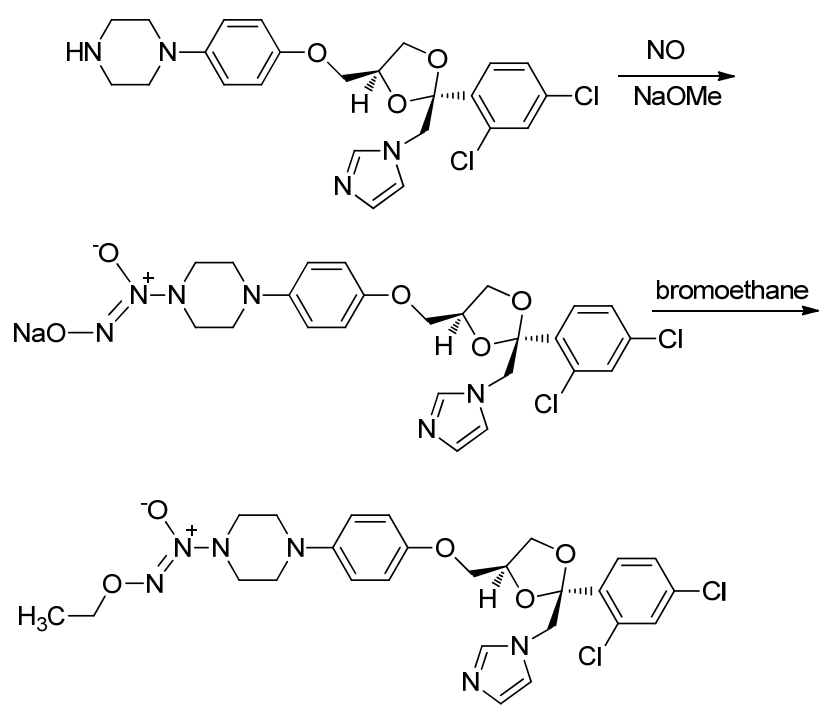

图式 27 带有 NO 供体的酮康唑的合成路线

Scheme 27 Synthetic routes of ketoconazole bearing NO donor

2008 年, Chowdhury 等 ${ }^{[67]}$ 合成了带有 NO 供体基团的 塞来昔布类似物. 该合成路线是先合成了塞来昔布类似 物, 再经过系列反应后与 $\mathrm{NO}$ 气体反应获得带有 $\mathrm{NO}$ 供体 基团的塞来昔布类似物(Eq. 16). 药理研究表明该产物的 抗炎活性比药物阿司匹林强, 但比药物塞来昔布弱.<smiles>CO[N+](=O)[O-]</smiles> 
2014 年, Abdellatif 等 ${ }^{[68]}$ 合成了带有 NO 供体基团的 塞来昔布类似物. 该合成路线是以溴代塞来昔布类似物 为原料与乙胺反应, 得到的产物与 $\mathrm{NO}$ 气体在四氢呋喃 和甲醇钠作用下生成带有 NO 供体基团的塞来昔布类似 物(Scheme 28). 药理研究发现与原药相比, NO 供体基 团的引入使其抗炎活性增强.

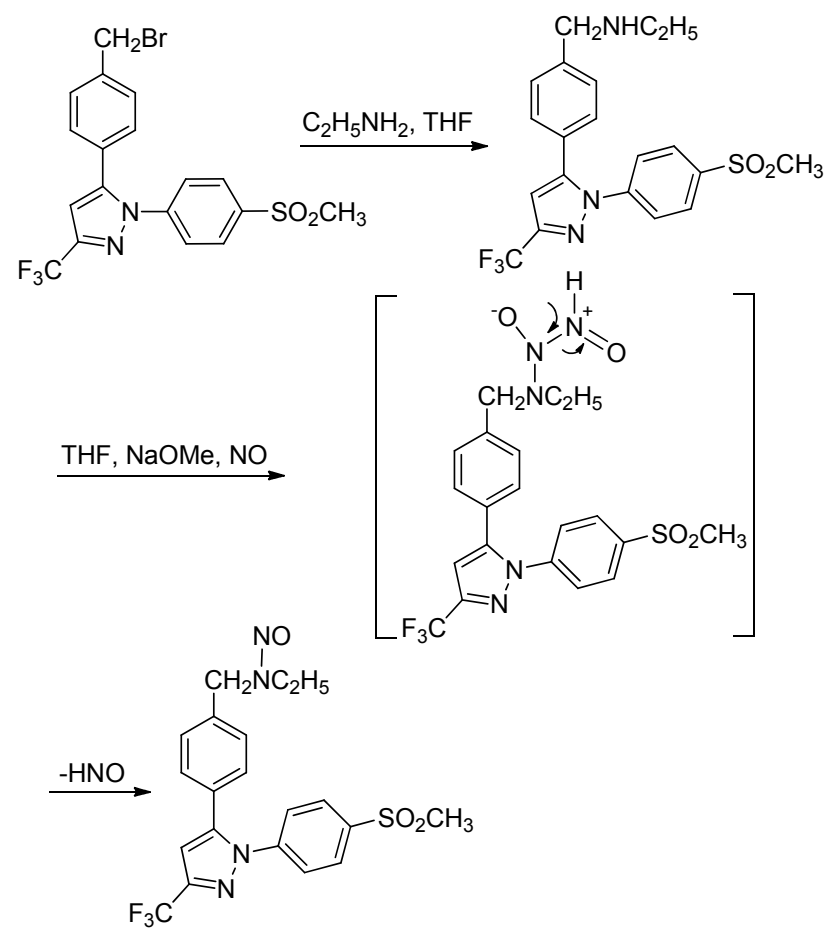

图式 28 带有 NO 供体的塞来昔布类似物的合成路线 Scheme 28 Synthetic routes of celecoxib analogue bearing NO donor

\section{$3.2 \mathrm{NH}_{3}$ 气体制备}

2010 年, Abdellatif 等 ${ }^{[69]}$ 合成了带有 NO 供体基团的 塞来昔布衍生物. 该合成路线是用二酮化合物 11 与 4甲基磺酰基苯基肼盐酸盐(12a)或 4-氨磺酰基苯基肼盐 酸盐(12b)反应生成相应的吡坐产物 $13 \mathrm{a}$ 或 13b. 将带有 硝基的吡唑衍生物进行经还原得到 $N$-羟氨基衍生物, 经 $\mathrm{NH}_{3}$ 气体和亚硝酸丁酯处理后再与溴乙酸乙酯反应 得到带有 NO 供体基团的塞来昔布衍生物(Scheme 29). 药理研究表明, 该化合物不仅具有抗炎活性, 而且还可 以降低非甾体抗炎药引起的副作用, 如胃刺激, 高血压 及血小板凝集.

\section{3 羧酸的 $\alpha-\mathrm{H}$ 取代}

2008 年, Abdellatif 等 ${ }^{[41]}$ 合成了带有 NO 供体基团的 丙烯酸衍生物. 该方法是直接将丙烯酸衍生物与偶氮二 醇烯鎓盐偶合获得带有 NO 供体基团的丙烯酸衍生物 (Eq. 17). 药理研究表明, 该化合物不仅具有良好的稳定 性, 而且其胃肠道不良反应明显降低.<smiles>[R]O[R16](=O)c1ccc(-n2nc(C(F)(F)F)cc2-c2ccc([N+](=O)[O-])cc2)cc1</smiles>

11a, 11b

$12 a, 12 b$<smiles>[R]O[SH](=O)(OC)c1ccc(-n2nc(C(F)(F)F)cc2-c2ccc(/[N+]([O-])=N/O[Na])cc2)cc1</smiles>

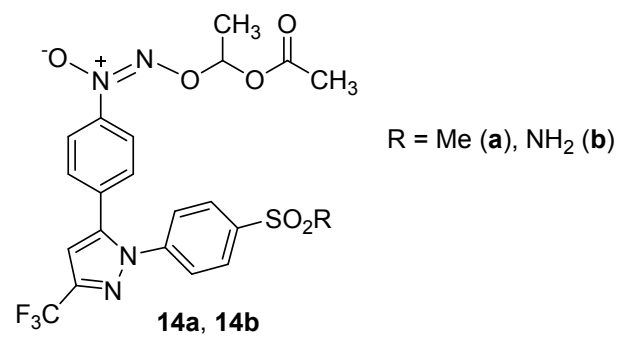

图式 29 带有 NO 供体的塞来昔布衍生物的合成路线 Scheme 29 Synthetic routes of celecoxib derivatives bearing NO donor

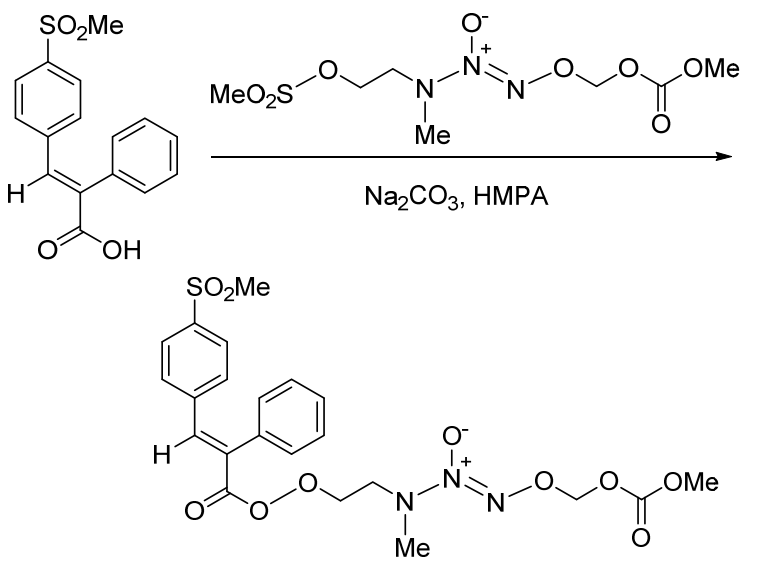

Abdellatif 等 ${ }^{[70]}$ 还用同样方法合成了带有 NO 供体 基团的塞来昔布类似物. 该合成路线以苯乙酮为原料合 成塞来昔布类似物，再与偶氮二醇烯鎓盐类 NO 供体基 团通过酯键连接. 药理研究表明该化合物在鼠血清中有 较好的 NO 释放能力, 但抗炎活性消失.

2008 年, Abdellatif 等 ${ }^{[71]}$ 合成了带有 NO 供体基团的 塞来昔布类似物. 该路线是用三氟甲苯丁二酮和 4-肼基 苯磺酰胺合成塞来昔布, 然后进行溴代, 得到的产物再 与偶氮二醇烯鎓盐类 NO 供体基团结合获得带有 NO 供 体基团的塞来昔布类似物(Eq. 18). 药理研究表明, 该化 合物具有较好的 NO 释放能力, 而且是高度选择性的 COX-2 抑制剂. 2011 年, Abdellatif 等 ${ }^{[72]}$ 用此方法合成了 
带有 NO 供体基团的罗非昔布衍生物. 该合成路线是用 2-溴-1-(4-甲磺酰基)苯乙酮与对甲基苯乙酸合成罗非昔 布类似物, 产物经过溴代反应后与 NO 供体基团发生成 酯反应获得带有 NO 供体基团的罗非昔布衍生物, 药理 研究表明, 该化合物可以选择性地抑制 COX-2 从而产 生抗炎作用, 而且还可以显著降低非甾体类抗炎药引起 的心血管不良反应.

2008 年, Velázquez 等 ${ }^{[73]}$ 合成了带有 NO 供体基团的 阿司匹林衍生物. 该合成路线是以吡咯烷-2-甲醇为原 料合成偶氮二醇烯鎓盐类 NO 供体, 再与氯代的阿司匹 林衍生物偶合获得带有 NO 供体基团的阿司匹林衍生物 (Eq. 19). 药理研究表明, 由于 NO 供体基团的引入使该 化合物的抗炎活性高于原药阿司匹林.

2012 年, Kaur 等 ${ }^{[42]}$ 合成了带有 NO 供体基团的美各
里替尼衍生物. 该合成路线利用四氢吡咯为原料合成偶 氮二醇烯鎓盐 NO 供体基团，以 5-氯-2-甲氧基苯甲酸为 原料合成药物美各里替尼, 再通过酰胺化将两者连接获 得带有 NO 供体基团的美各里替尼衍生物(Scheme 30). $\mathrm{NO}$ 释放研究表明该化合物具有较好的 NO 释放效应. 该化合物在治疗糖尿病的同时降低了心血管发生病变 的风险.

2015 年, $\mathrm{Xu}$ 等 ${ }^{[43]}$ 合成了带有 $\mathrm{NO}$ 供体基团的乙丙昔 罗衍生物. 该反应是用卤代的 NO 供体基团和乙丙昔罗 在 $\mathrm{Cs}_{2} \mathrm{CO}_{3}$ 存在的 DMF 中发生羧基的 $\alpha$ 位 $\mathrm{H}$ 取代反应 获得 NO 供体化合物(Eq. 20). NO 释放研究发现，该 NO 供体药物在体内能够比有机硝酸酯类 $\mathrm{NO}$ 供体更有效地 释放 NO，且不会产生药物耐受性.<smiles>CC(=O)OCO/N=[N+](\O)N1CCC[C@H]1C(=O)O</smiles><smiles>CC(=O)OCO/N=[N+](\[O-])N1CCC[C@H]1C(=O)OCc1ccc(-c2cc(C(F)(F)F)nn2-c2ccc(S(N)(=O)=O)cc2)cc1</smiles><smiles>CC(=O)OCO/N=C(\O)N1CCC[C@H]1C</smiles><smiles>CC(=O)Oc1ccccc1C(=O)OCCl</smiles><smiles>CC(=O)OCO/N=[N+](\[O-])N1CCC[C@H]1C(=O)OCOC(=O)c1ccccc1OC(C)=O</smiles>

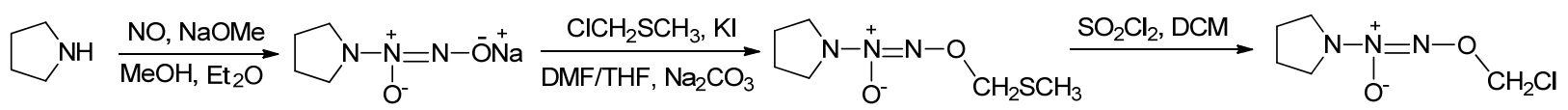<smiles>COc1ccc(Cl)cc1C(=O)NCCc1ccc(C(=O)OCO/N=[N+](\[O-])N2CCCC2)cc1</smiles>

图式 30 带有 NO 供体的美各里替尼衍生物的合成路线

Scheme 30 Synthetic routes of meglitinide derivative bearing NO donor<smiles>Cc1cc(C)cc(NC(=O)Cc2ccc(OC(C)(C)C(=O)O)cc2)c1</smiles><smiles>Cc1cc(C)cc(NC(=O)Cc2ccc(OC(C)(C)C(=O)OCO/N=[N+](\[O-])N3CCCC3)cc2)c1</smiles> 
2016 年, Gazzano 等 ${ }^{[44]}$ 合成了带有 NO 供体基团的 多柔比星衍生物. 该路线是将 NO 供体基团通过羧基的 $\alpha$ 位 $\mathrm{H}$ 取代反应连接到多柔比星骨架上获得 NO 供体化 合物. 药理研究表明, 该化合物的 NO 释放能力与连接 两个硝酸酯 NO 供体基团的化合物相当.

2016 年, $\mathrm{Xu}$ 等 ${ }^{[74]}$ 合成了带有 $\mathrm{NO}$ 供体的卵清素衍生 物. 该路线是将 $\mathrm{NO}$ 供体基团通过羧基的 $\alpha$ 位 $\mathrm{H}$ 取代反 应连接到卵清素骨架上获得 NO 供体化合物. 药理研究 表明, 所有目标化合物显示出有效的抗增殖活性, 而且 具有卵清素不具备的诱导细胞调亡并阻止 Bel-7402 细 胞 $\mathrm{S}$ 期的细胞周期的活性.

\section{4 酯化反应}

2009 年, Abdellatif 等 ${ }^{[17,75]}$ 用酯化反应合成了一系列 带有 NO 供体基团的非甾体类抗炎药. 该合成路线是将 非甾体类抗炎药(阿司匹林、吲哚美辛、塞来昔布和布 洛芬)经过系列反应生成酰氯, 再与偶氮二醇烯鎓盐 NO 供体基团通过酯键连接获得带有 NO 供体基团的非甾体 类抗炎药(Scheme 31). 药理研究表明, 这一系列化合物 具有 NO 释放效应, 不仅增强了抗炎作用而且还可以显
著降低胃肠道不良反应.

2016 年, Bertinaria 等 ${ }^{[56]}$ 合成了系列带有 NO 供体基 才的二氢青蒿素衍生物. 该路线是先进行亲核取代反应 将亚氨基连接到二氢青蒿素母体再与溴代的 NO 供体基 团发生取代获得 NO 供体化合物(Eq. 21). 药理研究表 明，该类化合物在体外和体内对抗伯氏症原虫实验中显 示出与青蒿琥酯和蒿甲醚相当的抗症原虫活性, 具有比 原药更高的活性.

\section{5 酰胺缩合}

2013 年, Kutty 等 ${ }^{[39]}$ 合成了带有 NO 供体基团的卤代 呋喃酮衍生物. 该合成路线是先用卤代呋喃酮衍生物与 丙二酸二酰氯反应，得到的产物再与偶氮二醇烯鎓盐类 NO 供体基团经酰胺缩合得到带有 NO 供体基团的卤代 呋喃酮衍生物(Eq. 22). 药理研究表明, 该化合物具有比 原药更强的抗菌活性. 而且由于 NO 供体基团的引入, 使其具有了双效抗菌效应.

2014 年, Smirnov 等 ${ }^{[76]}$ 合成了硝酸酯与偶氮二醇烯 鎓盐结合的化合物. 该合成路线是先用 $\mathrm{NO}$ 气体在甲醇

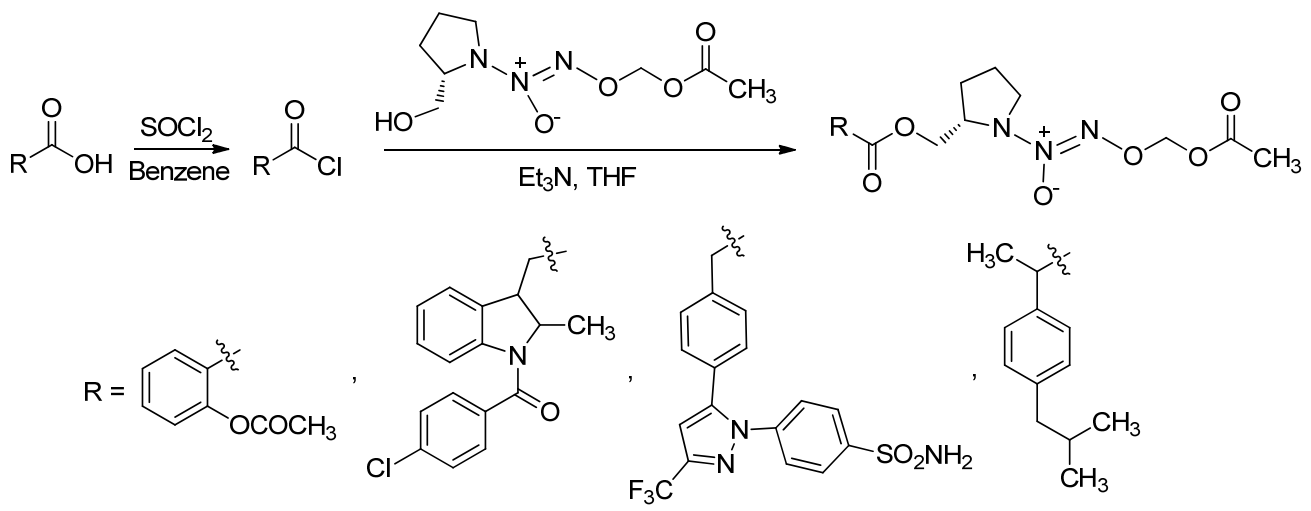

图式 31 带有 NO 供体的非甾体类抗炎药衍生物的合成路线

Scheme 31 Synthetic routes of NSAIDs derivatives bearing NO donor

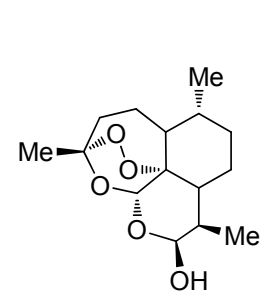<smiles>O=C(O)CCC(=O)OCO/N=[N+](\[O-])N1CCCC1</smiles>

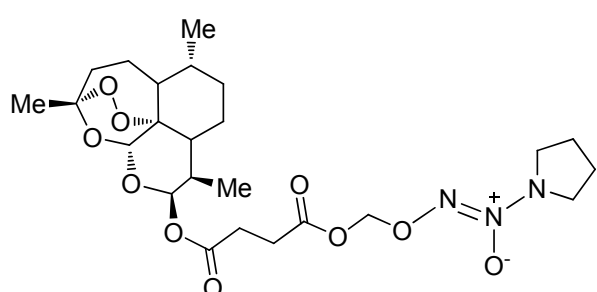


钠存在下合成偶氮二醇烯鎓盐, 再经取代反应与不饱和 烃连接得到带有双键的偶氮二醇烯鎓盐, 经加成反应加 溴后与 $\mathrm{AgNO}_{3}$ 发生取代得到目标化合物(Scheme 32). 此化合物显著地改变了 $\mathrm{NO}$ 释放速率. 此合成方法可以 同时引入多个 NO 供体基团, 改变 NO 在体内的释放速 率, 这可以使我们在一定范围内对 NO 供体化合物 NO 释放动力学进行调节.

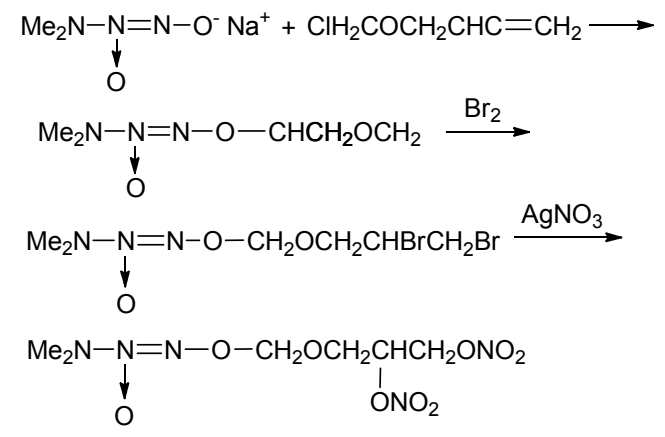

图式 32 偶氮二醇烯鎓盐与硝酸酯杂合物的合成路线 Scheme 32 Synthetic routes of azo-diol enenium salts with nitrate

综上所述，通过酯键将偶氮二醇烯鎓盐类 NO 供体 与药物进行连接是偶氮二醇烯鎓盐类 NO 供体药物较好 的合成途径, 此方法得到的目标化合物具有较高的产 率, 其反应过程简单, 无污染, 易于操作, 而且药物进 入体内经水解后可分别发挥各自的药理活性.

\section{4 肜类 NO 供体的合成}

2009 年, Abdel-Hafez 等 ${ }^{[21]}$ 合成了具有 NO 释放能力 的吡唑-3-羧酸衍生物 $15 \mathrm{a}$. 该合成路线是将吡唑-3-羧酸 进行酰氯化, 与对氨基苯丙酮偶合后经 $\mathrm{NH}_{2} \mathrm{OH}$ 处理后 获得具有 NO 释放能力的吡唑-3-羧酸衍生物(Eq. 23). 药理研究表明, 该化合物具有较好的 NO 释放能力, 同 时发现其抗水肿活性比药物吲哚美辛强.

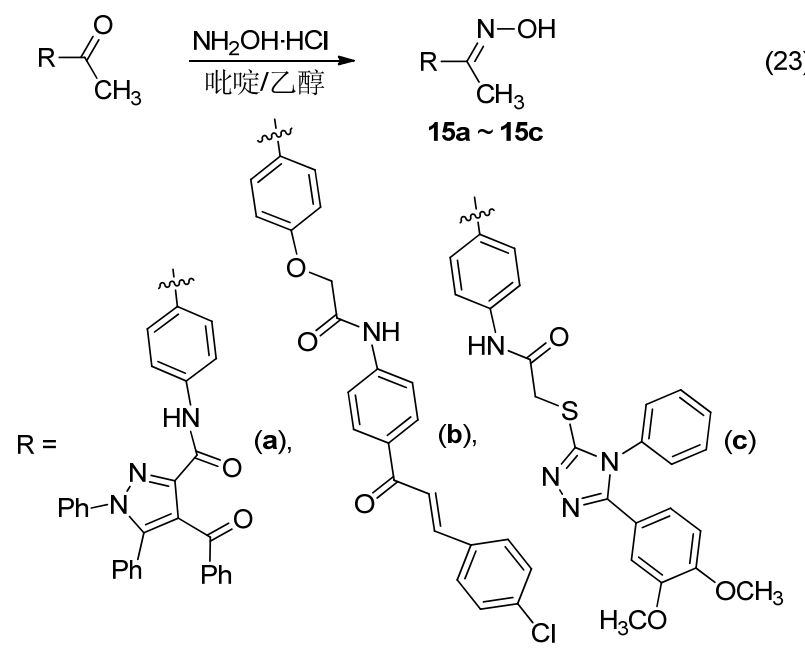

2012 年, Mourad 等 ${ }^{[35]}$ 合成了带有 NO 供体基团的查 尔酮衍生物 $15 \mathrm{~b}$. 该合成路线是先合成了查尔酮的溴代 衍生物, 再与对羟基苯丙酮反应, 获得的产物经 $\mathrm{NH}_{2} \mathrm{OH}$ 处理后得到带有肟类 NO 供体基团的查尔酮衍生物(Eq. 23). 药理研究发现, 该化合物的抗癌活性在加入 NO 供 体基团后减弱.

2014 年, Abuo-Rahma 等 ${ }^{[77]}$ 合成了能够释放 NO 的 三唑类化合物 $15 \mathrm{c}$. 该合成路线是用对氨基苯丙酮和溴 乙酰溴反应得到的产物与三唑化合物偶合，然后经 $\mathrm{NH}_{2} \mathrm{OH}$ 处理得到能够释放 $\mathrm{NO}$ 的三唑类化合物(Eq. 23). $\mathrm{NO}$ 释放研究发现，该化合物具有 $\mathrm{NO}$ 缓释作用，同时药 理研究表明该化合物具有抗炎及抗恶性细胞增殖的活 性.

2011 年, Shi 等 ${ }^{[53]}$ 合成了带有 NO 供体基团的司他 夫定衍生物. 该合成路线是以司他夫定为原料与丁二酸 酐反应, 得到的产物再与 NO 供体基团偶合获得带有 NO 供体基团的司他夫定衍生物(Eq. 24). 药理研究表 明，该化合物不仅具有比阿昔洛韦更好的抗病毒活性， 而且其毒副作用比阿昔洛韦弱.

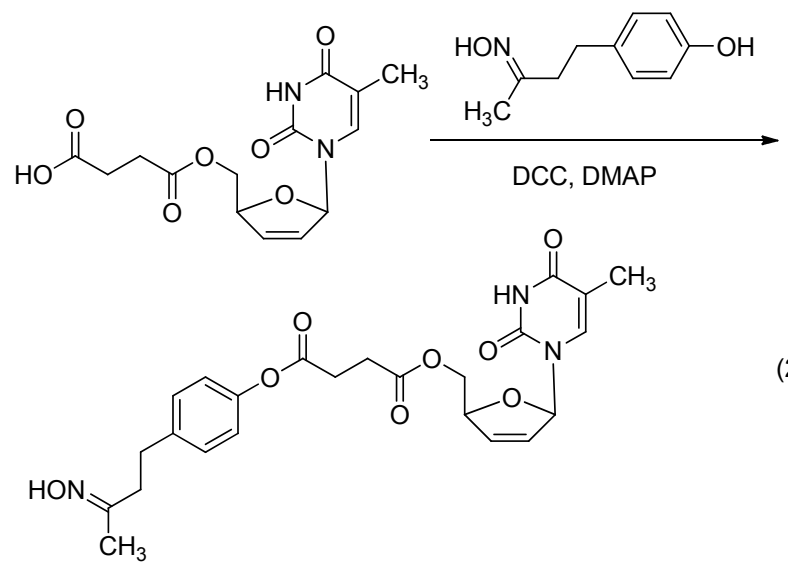

2016 年, Cassien 等 ${ }^{[78]}$ 合成了一系列具有不同芳族 取代基的新型杂化 2-(二乙氧基磷酰基)- $N$-(亚苄基)丙烷2-氧化胺衍生物(Scheme 33). 该类化合物具有肟结构, 能够释放 NO. 药理研究表明, 该类化合物不仅具有很 好的细胞毒活性, 而且对血管具有保护作用.

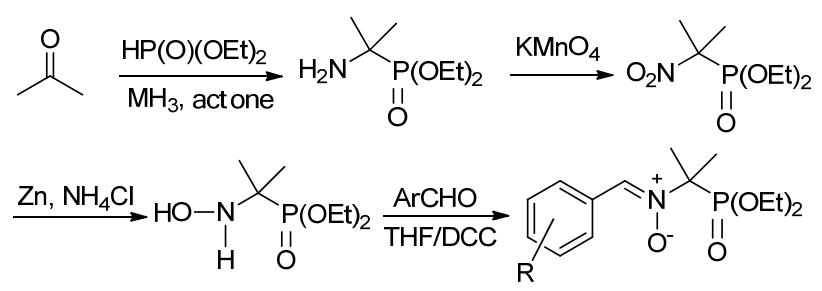

图式 $33 \beta$-磷酸化硝酮的合成路线

Scheme 33 Synthetic routes of $\beta$-phosphorylated nitrone 


\section{NO-金属配合物类 NO 供体的合成}

NO-金属配合物类 NO 供体主要是指金属元素与具 有 NO 释放效应的配位基形成的配合物. 主要代表药物 为硝普钠 ${ }^{[79]}$ ，临床上主要用于高血压危象患者的急救， 其在体内酶或硫醇作用下释放 NO 而舒张血管. 此类 NO 供体具有一些不足之处, 比如释放 NO 要依赖特定 的酶、温度、 $\mathrm{PH}$ 值等.

2013 年, Mcquilken 等 ${ }^{[80]}$ 合成了光致释放的 NO-金 属配合物类 NO 供体化合物(Scheme 34). 由于硫元素的 存在, 化合物 17 在光致释放时可以做到完全可逆, 以氮 元素连接的化合物 19 不可以.

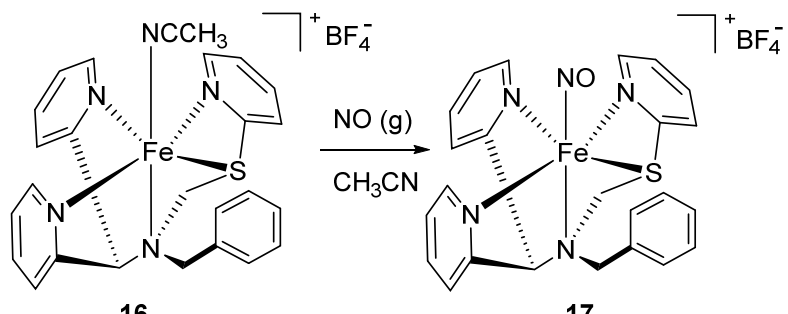

16

17

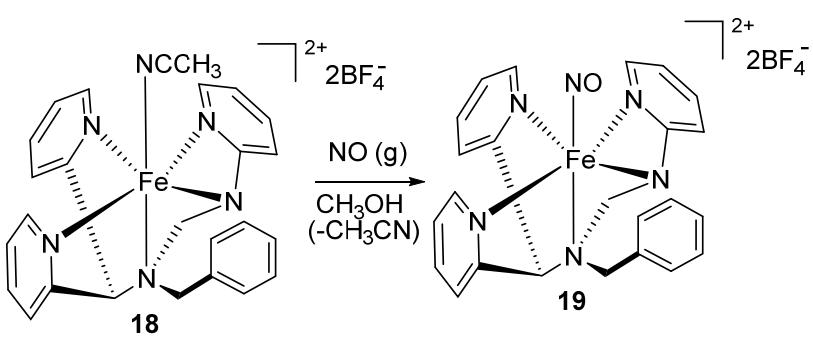

图式 $34\{\mathrm{FeNO}\}^{7}$ 铁配合物的合成

Scheme 34 Synthesis of $\{\mathrm{FeNO}\}^{7}$ complexes

2014 年, Sanina 等 ${ }^{[81]}$ 合成了 NO-金属配合物类 NO 供体四亚硝基双核铁配合物(Scheme 35). 与其他 NO 供 体化合物相比, 此化合物铁原子和 NO 分子的连接更加 稳定.

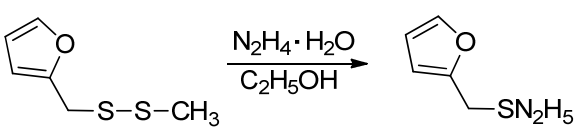

$$
\underset{\mathrm{Na}_{2} \mathrm{Fe}_{2}\left(\mathrm{~S}_{2} \mathrm{O}_{3}\right)_{2}(\mathrm{NO})_{3}}{\longrightarrow}\left[\mathrm{Fe}_{2}\left(\mathrm{SC}_{5} \mathrm{H}_{5} \mathrm{O}\right)_{2}(\mathrm{NO})_{4}\right]
$$

图式 35 四亚硝基双核铁配合物的合成

Scheme 35 Synthesis of tetranitrosyl binuclear iron complex

2016 年, Monti 等 ${ }^{[82]}$ 合成了镍-哌嗪/NO 供体化合物 20(图 1). 通过起始 $N$-氨基乙基哌嗪与水杨醛的缩合获 得该金属-壬酸盐的略微改性的形式. 金属-壬酸盐基于 具有壬酸基团的特定多胺, $N$-氨基乙基哌嗪的官能化, 并通过与金属离子络合而稳定化, 通过与水反应(每个 络合物 2 个 $\mathrm{NO}$ 分子) 以金属, $\mathrm{pH}$ 和温度依赖性方式释放
NO. 药理研究表明，该化合物只对高血压小鼠有很好 的降压作用，对正常小鼠血压无影响. 由于其突出的血 管扩张活性而被认为是抗高血压治疗的潜在候选药物.

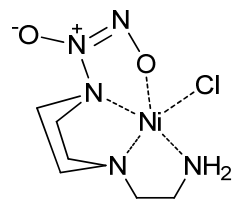

20

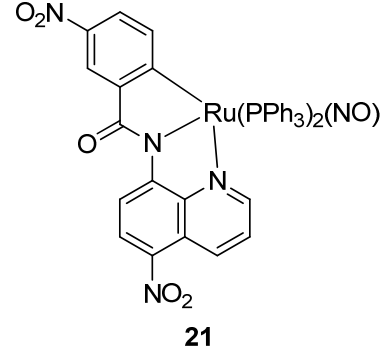

21
图 1 NO-金属配合物

Figure 1 NO-metal complexes

2016 年, Sakhaei 等 ${ }^{[83]}$ 合成了 NO-金属配合物类 NO 供体化合物 NO-铜配合物(Eq. 25). 与其他 NO 供体化合 物相比，此化合物通过氧原子从亚硝酸盐在铜(II)位点 转移而释放 NO, 这是从未报道过的 NO 释放新途径.
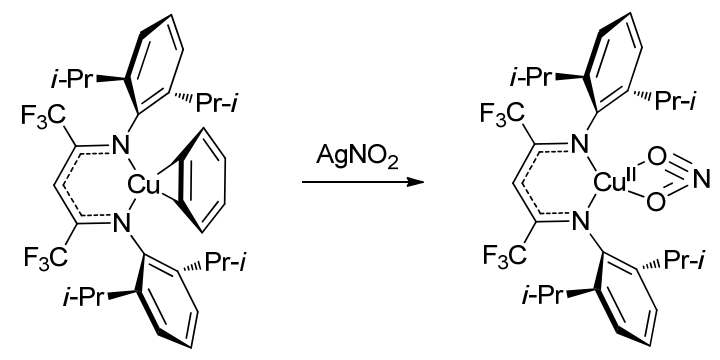

2016 年, Kumar 等 ${ }^{[84]}$ 合成了 NO-金属配合物类 NO 供体化合物 21(图 1). 该路线通过从具有酰胺键的二齿 配体衍生的 $\mathrm{C}-\mathrm{H}$ 键活化来合成新的 $\sigma$-芳基钉(III)络合 物, 将这些有机金属钉(III)络合物用 NO 处理, 得到亚 硝酰基络合物. NO 释放研究表明, 此化合物具有光致 释放特性, 可以实现 NO 的靶向释放和定量释放, 对于 NO 的缓释控释具有巨大意义.

\section{$6 S$-亚硝基硫醇类 NO 供体的合成}

$S$-亚硝基硫醇在生物体内广泛存在，是蛋白、多肽、 硫醇等颈基亚硝基化的产物, 参与 $\mathrm{NO}$ 的储存和转运, 其可在生理条件下自发释放 $\mathrm{NO}$, 热、光、碱性 $\mathrm{pH}$ 以及 与 $\mathrm{O}_{2}$ 接触可促进其反应 ${ }^{[85]}$. $\mathrm{NO}$ 可以与硫醇类物质的颈 基以共价键的方式结合形成 RSNO, 使其性质相对稳定. 外源性 RSNO 可通过转亚硝基反应把 NO 转给体内的硫 醇, 最终实现内源性 NO 的转运. $S$-亚硝基硫醇是 NO 在 体内发挥生物效应的基本形式, 主要代表性化合物为 $S$ 亚硝基谷胱甘肽 ${ }^{[86]}$.

2008 年, Stasko 等 ${ }^{[87]}$ 合成了具有 NO 释放能力的 $S$ 亚硝基硫醇修饰的树状大分子. 这种树状大分子设计增 
大了 $\mathrm{NO}$ 的储运量, 可以使 $\mathrm{NO}$ 在体内更有效地释放. 药理研究表明, 该化合物具有抗菌效应和促进伤口愈合 的作用. Stasko 等设计了两条合成路线.

合成路线 1 是以 $N$-乙酰- $D / L$-青霉胺为原料经系列 反应与树状大分子 G4-PAMAM 结合后再经 $\mathrm{NaNO}_{2}$ 处理 得到具有 $\mathrm{NO}$ 释放能力的 $S$-亚硝基硫醇修饰的树状大分 子(Scheme 36).

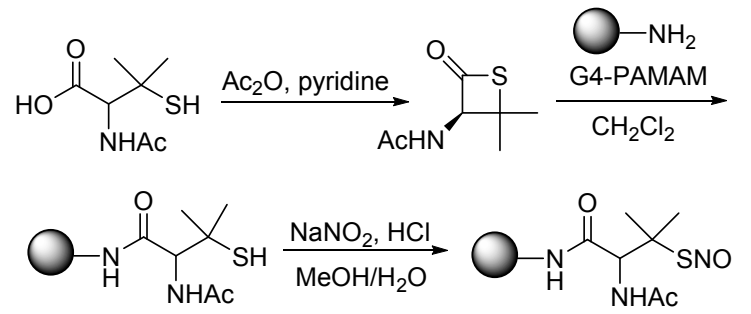

图式 $36 S$-亚硝基硫醇修饰的树状大分子 G4-SNAP 的合成 Scheme 36 Synthesis of $S$-nitrosothiol modified generation 4 PAMAM dendrimer, G4-SNAP

合成路线 2 是以 $N$-乙酰半胱氨酸为原料, 经系列反 应与树状大分子 G4-PAMAM 结合, 再经亚硝酸异戊酯 处理得到具有 $\mathrm{NO}$ 释放能力的 $S$-亚硝基硫醇修饰的树状 大分子(Scheme 37).
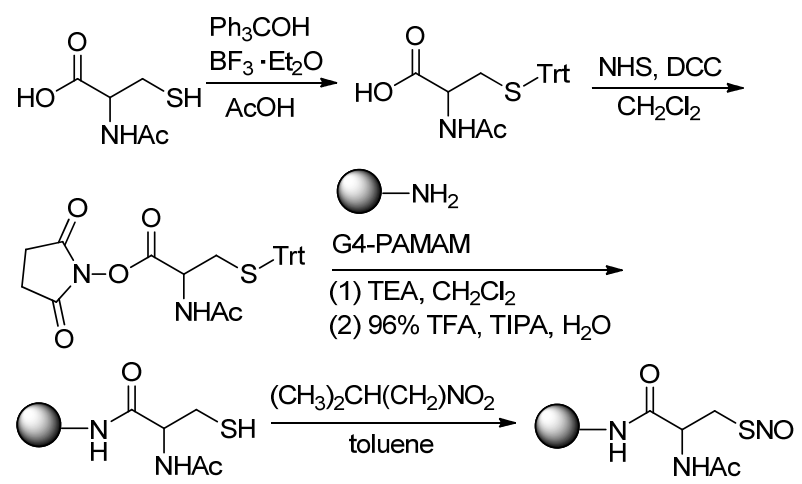

图式 $37 S$-亚硝基硫醇修饰的树状大分子 G4-NACysNO 的合 成

Scheme 37 Synthesis of G4-NACysNO, S-nitrosothiol modified generation 4 PAMAM dendrimer

2009 年, Llop 等 ${ }^{[88]}$ 合成了用于正电子发射断层扫描 显影的 NO 供体化合物. 该反应是以谷胱甘肽为原料合 成亚硝基谷胱甘肽(Eq. 26). 该路线采用放射性亚硝基 化技术，可以在短时间内反应得到大量产物.

\section{7 其他类 NO 供体}

除以上所述类型的 $\mathrm{NO}$ 供体外, 还存在多种类型的 $\mathrm{NO}$ 供体 ${ }^{[16]}$ (图 2): (1)斯德酮亚胺类 NO 供体属于介离子 类杂环化合物, 在生理环境下可自发降解或者经体内酶 解后释放 NO, 具有抗血小板凝集、抗血栓和扩张血管

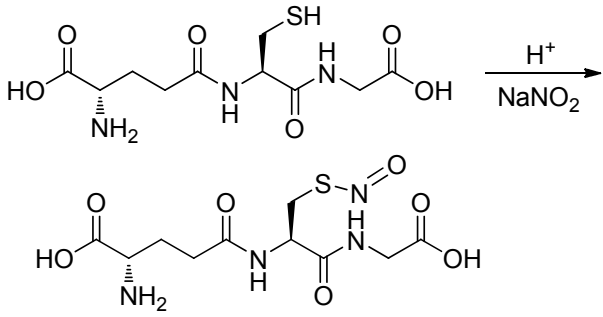

等生物活性, 代表性化合物为 SIN-1 ${ }^{[89]}$. (2)胍类 NO 供 体, 在体内生理环境下不能自发释放 NO, 必须在体内 的 P450 或 NOS 等酶的催化下经多步代谢才能生成 NO, 常具有抗白血病及其他肿瘤的活性，代表性化合物有 $N$-hydroxydebrisoquine ${ }^{[90]}$ 等. (3) $N$-差基- $N$-亚硝基胺类 $\mathrm{NO}$ 供体通常比较稳定, 需要在体内经过氧化物酶作用 后释放 NO, 代表性化合物有 Alanosine ${ }^{[9]]}$ 等. (4) $N$-羟基 腿类、肽偶联 NONOates 类、C-亚硝基烃类等 NO 供体. 由于近 10 年来这几类 $\mathrm{NO}$ 供体化合物合成方法的相关 研究较少, 本文不再进行综述.

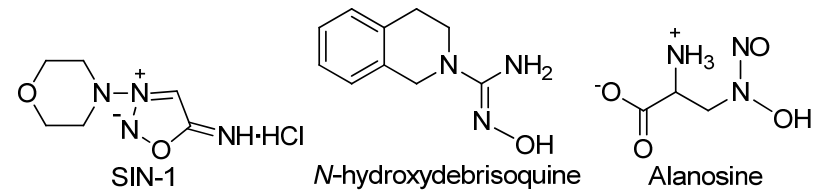

图 2 其他类 $\mathrm{NO}$ 供体药物

Figure 2 Other types of NO donor drugs

\section{8 总结}

综上所述, NO 供体化合物研究在近几年已得到较 大的发展，已合成了多种多样的带有不同类型 NO 供体 基团的化合物. NO 供体化合物因其良好的生物活性, 较低的不良反应，在医药卫生领域的应用越来越广泛. 因此, 采用化学方法合成该类化合物具有十分重要的意 义。

本文对近 10 年文献中报道的 NO 供体化合物的合 成方法进行了综述，说明了 NO 供体化合物的合成具有 多样的反应类型和灵活性的反应体系. 其中, 应用较为 广泛的是制成酯类前药的设计方案. 现已合成的 NO 供 体化合物具有结构简单、易于合成、能够降低药物不良 反应、提高药效等优点, 如呋咱氮氧化物类 NO 供体具 有更广泛的生物活性，在体内游离颈基和颈基阴离子作 用下均可释放 NO, 而且不易产生耐受性. 偶氮二醇烯 鎓盐类 NO 供体偶联特定的取代基团可延长其半衰期, 与某些特定载体(如酶的底物、单克隆抗体等)偶联可制 成具有靶向缓释 $\mathrm{NO}$ 作用的 $\mathrm{NO}$ 供体化合物. NO-金属卟 啉类 $\mathrm{NO}$ 供体还可以做到在肿瘤细胞定点释放 $\mathrm{NO}$ 来产 生抗癌活性. 但其亦具有易产生耐受性、稳定性差、无 靶向性、释放不可控等缺点, 所以限制了其在临床的应 
用, 有进一步研究的必要. 可以相信, 随着 NO 供体化 合物研究的进一步深入, 其合成方法必将不断推新, 会 开发出更多安全、高效、低毒的候选药物.

\section{References}

[1] Azizzadeh, B.; Yip, H. T.; Blackwell, K. E.; Horvath, S.; Calcaterra, T. C.; Buga, G. M. Laryngoscope 2001, 111, 1896.

[2] Huerta, S.; Chilka, S.; Bonavida, B. Int. J. Oncol. 2008, 33, 909.

[3] Agurla, S.; Gayatri, G.; Raghavendra, A. S. Nitric Oxide 2014, 43, 89.

[4] Jin, R. C.; Loscalzo, J. J. Blood Med. 2010, 2010, 147.

[5] Esplugues, J. V. Br.J. Pharmacol. 2002, 135, 1079.

[6] Wallace, J. L. Mem. Inst. Oswaldo Cruz 2005, 100(suppl. 1), 5.

[7] David, H.; Tracy, R. J. Pharm. Pharmacol. 2007, 59, 3.

[8] Afshar, J. K.; Pluta, R. M.; Boock, R. J.; Thompson, B. G.; Oldfield, E. H. J. Neurosurg. 1995, 83, 118.

[9] Rajagopalan, S.; Harrison, D. G. Circulation 1996, 94, 240.

[10] Steudel, W.; Scherrer-Crosbie, M.; Bloch, K. D.; Weimann, J.; Huang, P. L.; Jones, R. C. J. Clin. Invest. 1998, 101, 2468.

[11] Suzuki, H.; Shimosegawa, T.; Ohara, S.; Toyota, T. J. Gastroenterol. 1999, 34, 172.

[12] Kuo, P. C.; Schroeder, R. A. Ann. Surg. 1995, 221, 220.

[13] Bradley, S. A.; Steinert, J. R. J. Neurosci. Methods 2015, 245, 116.

[14] Serafim, R. A.; Primi, M. C.; Trossini, G. H. Curr. Med. Chem. 2012, 19, 386

[15] Carradori, S.; Mollica, A.; Monte, C. Molecules 2015, 20, 5667.

[16] Bian, H. Y. Ph.D. Dissertation, Shandong University, Shandong, 2011 (in Chinese) (边海勇, 博士论文, 山东大学, 山东, 2011.)

[17] Abdellatif, K. R.; Chowdhury, M. A.; Dong, Y.; Das, D.; Yu, G.; Velazquez, C. A.; Suresh, M. R.; Knaus, E. E. Bioorg. Med. Chem. Lett. 2009, 19, 3014.

[18] Abuo-Rahma, E. D. A. A.; Abdel-Aziz, M.; Mai, A. E. M.; Farag, H. H. Bioorg. Med. Chem. 2012, 20, 195.

[19] Luo, G.; Chen, Y. Y. Chin. J. New Drugs 2010, 19, 1322 (in Chinese).

(罗刚, 陈宇瑛, 中国新药杂志, 2010, 19, 1322.)

[20] López, G. V.; Blanco, F.; Hernández, P.; Ferreira, A.; Piro, O. E.; Batthyány, C.; González, M.; Rubbo, H.; Cerecetto, H. Bioorg. Med. Chem. 2007, 15, 6262.

[21] Abdel-Hafez, E. S. M. N.; Abuo-Rahma, E. D. A. A.; Abdel-Aziz, M.; Radwan, M. F.; Farag, H. Bioorg. Med. Chem. 2009, 17, 3829.

[22] Rolando, B.; Filieri, A.; Chegaev, K.; Lazzarato, L.; Giorgis, M.; De Nardi, C.; Fruttero, R.; Martel, S.; Carrupt, P. A.; Gasco, A. Bioorg. Med. Chem. 2012, 20, 841.

[23] Digiacomo, M.; Martelli, A.; Testai, L.; Lapucci, A.; Breschi, M. C.; Calderone, V.; Rapposelli, S. Bioorg. Med. Chem. 2015, 23, 422.

[24] Konter, J.; Mollmann, U.; Lehmann, J. Bioorg. Med. Chem. 2008, 16,8294 .

[25] Bertinaria, M.; Rolando, B.; Giorgis, M.; Montanaro, G.; Marini, E.; Collino, M.; Benetti, E.; Daniele, P. G.; Fruttero, R.; Gasco, A. Eur. J. Med. Chem. 2012, 54, 103.

[26] Biava, M.; Battilocchio, C.; Poce, G.; Alfonso, S.; Consalvi, S.; Di Capua, A.; Calderone, V.; Martelli, A.; Testai, L.; Sautebin, L.; Rossi, A.; Ghelardini, C.; Di Cesare Mannelli, L.; Giordani, A.; Persiani, S.; Colovic, M.; Dovizio, M.; Patrignani, P.; Anzini, M. Bioorg. Med. Chem. 2014, 22, 772.

[27] Liu, W.; Liu, C.; Gong, C.; Lin, W.; Guo, C. Bioorg. Med. Chem. Lett. 2009, 19, 1647.
[28] Peng, S. M.; Zou, X. Q.; Ding, H. L.; Ding, Y. L.; Lin, Y. B. Bioorg. Med. Chem. Lett. 2009, 19, 1264

[29] Dong, X.; Du, L.; Pan, Z.; Liu, T.; Yang, B.; Hu, Y. Eur. J. Med. Chem. 2010, 45, 3986.

[30] Zou, X. Q.; Peng, S. M.; Hu, C. P.; Tan, L. F.; Yuan, Q.; Deng, H. W.; Li, Y. J. Bioorg. Med. Chem. 2010, 18, 3020.

[31] Bai, R.; Yang, X.; Zhu, Y.; Zhou, Z.; Xie, W.; Yao, H.; Jiang, J.; Liu, J.; Shen, M.; Wu, X.; Xu, J. Bioorg. Med. Chem. 2012, 20, 6848.

[32] Wang, Q. Q.; Cheng, N.; Zheng, X. W.; Peng, S. M.; Zou, X. Q. Bioorg. Med. Chem. 2013, 21, 4301.

[33] Bhandari, S. V.; Bothara, K. G.; Patil, A. A.; Chitre, T. S.; Sarkate, A. P.; Gore, S. T.; Dangre, S. C.; Khachane, C. V. Bioorg. Med. Chem. 2009, 17, 390

[34] Bhandari, S. V.; Dangre, S. C.; Bothara, K. G.; Patil, A. A.; Sarkate, A. P.; Lokwani, D. K.; Gore, S. T.; Deshmane, B. J.; Raparti, V. T.; Khachane, C. V. Eur. J. Med. Chem. 2009, 44, 4622.

[35] Mourad, M. A.; Abdel-Aziz, M.; Abuo-Rahma Gel, D.; Farag, H. H. Eur. J. Med. Chem. 2012, 54, 907.

[36] Fang, L.; Feng, M.; Chen, F. Bioorg. Med. Chem. 2016, 24, 4611.

[37] Zhang, Y. C.; Zhou, J. P.; Wu, X. M.; Pan, W. H. Chin. Chem. Lett. 2009, 20, 302.

[38] Tamboli, Y.; Lazzarato, L.; Marini, E.; Guglielmo, S.; Novelli, M.; Beffy, P.; Masiello, P.; Fruttero, R.; Gasco, A. Bioorg. Med. Chem. Lett. 2012, 22, 3810.

[39] Kutty, S. K.; Barraud, N.; Pham, A.; Iskander, G.; Rice, S. A.; Black, D. S.; Kumar, N. J. Med. Chem. 2013, 56, 9517.

[40] Huang, Q.; Rui, E. Y.; Cobbs, M.; Dinh, D. M.; Gukasyan, H. J.; Lafontaine, J. A.; Mehta, S.; Patterson, B. D.; Rewolinski, D. A.; Richardson, P. F.; Edwards, M. P. J. Med. Chem. 2015, 58, 2821.

[41] Abdellatif, K. R.; Chowdhury, M. A.; Dong, Y.; Chen, Q. H.; Knaus, E. E. Bioorg. Med. Chem. 2008, 16, 3302.

[42] Kaur, J.; Bhardwaj, A.; Huang, Z.; Narang, D.; Chen, T. Y.; Plane, F.; Knaus, E. E. J. Med. Chem. 2012, 55, 7883.

[43] Xu, G. G.; Deshpande, T. M.; Ghatge, M. S.; Mehta, A. Y.; Omar, A. S.; Ahmed, M. H.; Venitz, J.; Abdulmalik, O.; Zhang, Y.; Safo, M. K. Biochemistry 2015, 54, 7178 .

[44] Gazzano, E.; Chegaev, K.; Rolando, B. Bioorg. Med. Chem. 2016, $24,967$.

[45] Fang, L. A. D.; Decker, M.; Kiehntopf, M.; Roegler, C.; Deufel, T.; Fleck, C.; Peng, S. X.; Zhang, Y. H.; Lehmann, J. J. Med. Chem. 2008, $51,4$.

[46] Chowdhury, M. A.; Abdellatif, K. R.; Dong, Y.; Yu, G.; Huang, Z.; Rahman, M.; Das, D.; Velazquez, C. A.; Suresh, M. R.; Knaus, E. E. Bioorg. Med. Chem. Lett. 2010, 20, 1324.

[47] Bertinaria, M.; Guglielmo, S.; Rolando, B.; Giorgis, M.; Aragno, C.; Fruttero, R.; Gasco, A.; Parapini, S.; Taramelli, D.; Martins, Y. C.; Carvalho, L. J. Eur. J. Med. Chem. 2011, 46, 1757.

[48] Zhao, J.; Prosser, K. E.; Chang, S. W. Dalton Trans. 2016, 45, 18079.

[49] Csont, T.; Ferdinandy, P. Pharmacol. Ther. 2005, 105, 57.

[50] Feelisch, M.; Schonafinger, K.; Noack, E. Biochem. Pharmacol. 1992, 44, 1149.

[51] Chen, L. Z. Y.; Kong, X. W.; Lan, E. D.; Huang, Z. J.; Peng, S. X.; Kaufman, D. L.; Tian, J. J. Med. Chem. 2008, 51, 4834.

[52] Ling, Y.; Ye, X.; Ji, H.; Zhang, Y.; Lai, Y.; Peng, S.; Tian, J. Bioorg. Med. Chem. 2010, 18, 3448.

[53] Shi, J. B.; Xu, S.; Wang, Y. P.; Li, J. J.; Yao, Q. Z. Chin. Chem. Lett. 2011, 22, 899

[54] Tang, W.; Xie, J.; Xu, S.; Lv, H.; Lin, M.; Yuan, S.; Bai, J.; Hou, Q.; Yu, S. J. Med. Chem. 2014, 57, 7600.

[55] Dos Santos, J. L.; Lanaro, C.; Chelucci, R. C.; Gambero, S.; 
Bosquesi, P. L.; Reis, J. S.; Lima, L. M.; Cerecetto, H.; Gonzalez, M.; Costa, F. F.; Chung, M. C. J. Med. Chem. 2012, 55, 7583.

[56] Bertinaria, M.; Orjuelasanchez, P.; Marini, E. J. Med. Chem. 2015, 58,7895 .

[57] Bian, H.; Feng, J.; Li, M.; Xu, W. Bioorg. Med. Chem. Lett. 2011, 21, 7025 .

[58] Borretto, E.; Lazzarato, L.; Spallotta, F.; Cencioni, C.; D'Alessandra, Y.; Gaetano, C.; Fruttero, R.; Gasco, A. ACS Med. Chem. Lett. 2013, 4, 994.

[59] Lu, M.-D.; Zhou, X.; Yu, Y.-J.; Li, P.-H.; Sun, W.-J.; Zhao, C.-G.; Zheng, Z.-Q.; You, T.; Wang, F.-H. Chin. Chem. Lett. 2013, 24, 415.

[60] Ai, Y.; Kang, F.; Huang, Z.; Xue, X.; Lai, Y.; Peng, S.; Tian, J.; Zhang, Y. J. Med. Chem. 2015, 58, 2452.

[61] Duan, W.; Li, J.; Inks, E. S.; Chou, C. J.; Jia, Y.; Chu, X.; Li, X.; Xu, W.; Zhang, Y. J. Med. Chem. 2015, 58, 4325.

[62] Massarico Serafim, R. A.; Goncalves, J. E.; de Souza, F. P.; de Melo Loureiro, A. P.; Storpirtis, S.; Krogh, R.; Andricopulo, A. D.; Dias, L. C.; Ferreira, E. I. Eur. J. Med. Chem. 2014, 82, 418.

[63] Lakshman, T. R.; Deb, J.; Paine, T. K. Dalton Trans. 2016, 45, 14053.

[64] Davies, K. M.; Wink, D. A.; Saavedra, J. E.; Keefer, L. K. J. Am. Chem. Soc. 2001, 32, 5473.

[65] Smith, D. J.; Chakravarthy, D.; Pulfer, S.; Simmons, M. L.; Hrabie, J. A.; Citro, M. L. J. Med. Chem. 1996, 39, 1148.

[66] Chakrapani, H.; Goodblatt, M. M.; Udupi, V.; Malaviya, S.; Shami, P. J.; Keefer, L. K.; Saavedra, J. E. Bioorg. Med. Chem. Lett. 2008, 18,950 .

[67] Chowdhury, M. A.; Abdellatif, K. R.; Dong, Y.; Knaus, E. E. Bioorg. Med. Chem. 2008, 16, 8882.

[68] Abdellatif, K. R.; Moawad, A.; Knaus, E. E. Bioorg. Med. Chem. Lett. 2014, 24, 5015.

[69] Abdellatif, K. R.; Chowdhury, M. A.; Velazquez, C. A.; Huang, Z.; Dong, Y.; Das, D.; Yu, G.; Suresh, M. R.; Knaus, E. E. Bioorg. Med. Chem. Lett. 2010, 20, 4544.

[70] Abdellatif, K. R.; Chowdhury, M. A.; Dong, Y.; Knaus, E. E. Bioorg. Med. Chem. 2008, 16, 6528.

[71] Abdellatif, K. R.; Chowdhury, M. A.; Dong, Y.; Velazquez, C.; Das, D.; Suresh, M. R.; Knaus, E. E. Bioorg. Med. Chem. 2008, 16,
9694.

[72] Abdellatif, K. R.; Huang, Z.; Chowdhury, M. A.; Kaufman, S.; Knaus, E. E. Bioorg. Med. Chem. Lett. 2011, 21, 3951.

[73] Velázquez, C. A.; Chen, Q. H.; Citro, M. L.; Keefer, L. K.; Knaus, E. E. J. Med. Chem. 2008, 51, 1954.

[74] Xu, S.; Wang, G.; Yan, L. Bioorg. Med. Chem. Lett. 2016, 26, 2795.

[75] Abdellatif, K. R.; Chowdhury, M. A.; Dong, Y.; Das, D.; Yu, G.; Velazquez, C.; Suresh, M. R.; Knaus, E. E. Bioorg. Med. Chem. 2009, 17, 5182.

[76] Smirnov, G. A.; Gordeev, P. B.; Nikitin, S. V. Russ. Chem. Bull. 2014, 63, 487.

[77] Abuo-Rahma, E. D. A. A.; Abdel-Aziz, M.; Beshr, E. A.; Ali, T. F. Eur. J. Med. Chem. 2014, 71, 185.

[78] Cassien, M.; Petrocchi, C.; Thétiot-Laurent, S. Eur. J. Med. Chem. 2016, 119, 197.

[79] Vogt, M. A.; Vogel, A. S.; Pfeiffer, N.; Gass, P.; Inta, D. Eur. Neuropsychopharmacol. 2015, 25, 1848.

[80] Mcquilken, A. C.; Yang, H.; Sutherlin, K. D.; Siegler, M. A.; Hodgson, K. O.; Britt, H. J. Am. Chem. Soc. 2013, 135, 14024.

[81] Sanina, N. A.; Kozub, G. I.; Kondratéva, T. A.; Korchagin, D. V.; Shilov, G. V.; Emelýanova, N. S. J. Mol. Struct. 2014, 1075, 159.

[82] Monti, M.; Ciccone, V.; Pacini, A. Pharmacol. Res. 2016, 107, 352.

[83] Sakhaei, Z.; Kundu, S.; Donnelly, J. Chem. Commun. 2017, 53, 549.

[84] Kumar, R.; Kumar, S.; Bala, M. RSC Adv. 2016, 6, 72096.

[85] Grossi, L.; Montevecchi, P. C. J. Org. Chem. 2002, 67, 8625.

[86] Kumari, S.; Sammut, I. A.; Giles, G. I. Eur. J. Med. Chem. 2014, $737,168$.

[87] Stasko, N. A.; Fischer, T. H.; Schoenfisch, M. H. Biomacromolecules 2008, 9, 834.

[88] Llop, J.; Gómez-Vallejo, V.; Bosque, M.; Quincoces, G.; Peñuelas, I. Appl. Radiat. Isot. 2009, 67, 95.

[89] Priora, R.; Margaritis, A.; Frosali, S.; Coppo, L.; Summa, D.; Giuseppe, D. D. Pharmacol. Res. 2011, 64, 289.

[90] Ming, X.; Noriko, F.; Zhong, W.; Tingwei, C.; Satoshi, K.; Janczuk, A. J. Bioorg. Med. Chem. 2002, 10, 3049.

[91] Tyagi, A. K.; Cooney, D. A. Adv. Pharmacol. Chemother. 1984, 20, 69. 